NYU-TH-05/12/10

\title{
Perturbations of Self-Accelerated Universe
}

\author{
Cédric Deffayet $^{a, b}$, Gregory Gabadadze ${ }^{c}$ and Alberto Iglesias ${ }^{c}$ \\ ${ }^{a} A P C^{1}, 11$ place Marcelin Berthelot, \\ 75005 Paris Cedex 05, France \\ ${ }^{b}$ GReCO/IAP ${ }^{2}, 98$ bis Boulevard Arago, \\ 75014 Paris, France \\ ${ }^{c}$ Center for Cosmology and Particle Physics \\ Department of Physics, New York University, New York, NY, 10003, USA
}

\begin{abstract}
We discuss small perturbations on the self-accelerated solution of the DGP model, and argue that claims of instability of the solution that are based on linearized calculations are unwarranted because of the following: (1) Small perturbations of an empty self-accelerated background can be quantized consistently without yielding ghosts. (2) Conformal sources, such as radiation, do not give rise to instabilities either. (3) A typical non-conformal source could introduce ghosts in the linearized approximation and become unstable, however, it also invalidates the approximation itself. Such a source creates a halo of variable curvature that locally dominates over the self-accelerated background and extends over a domain in which the linearization breaks down. Perturbations that are valid outside the halo may not continue inside, as it is suggested by some non-perturbative solutions. (4) In the Euclidean continuation of the theory, with arbitrary sources, we derive certain constraints imposed by the second order equations on first order perturbations, thus restricting the linearized solutions that could be continued into the full nonlinear theory. Naive linearized solutions fail to satisfy the above constraints. (5) Finally, we clarify in detail subtleties associated with the boundary conditions and analytic properties of the Green's functions.
\end{abstract}

\footnotetext{
${ }^{1}$ UMR 7164 (CNRS, Université Paris 7, CEA, Observatoire de Paris)
}

${ }^{2}$ UMR 7095 (CNRS, Université Paris 6) 


\section{Introduction and summary}

The accelerated expansion of the Universe could be due to modification of gravity at cosmological distance scales. One "existence proof" for such a scenario is the DGP model [1, in which the self-accelerated solution [2] can be used to describe the observed speed-up of the cosmic expansion [3].

While there are a number of interesting works exploring the consistency of the self-accelerated solution with the data, see, e.g. [3]-[19], the linearized perturbations on this background were claimed to contain a ghost-like mode [20, 21, 22, 23, 24].

If the results of the linearized calculations were reliable, this would exclude the self-accelerated background from a set of physically acceptable solutions of the DGP model. However, we will show below that the issue is more involved. Small perturbations on an empty self-accelerated background can be quantized without yielding ghosts. Moreover, for conformal sources, such as radiation, the small perturbations are still ghost free. Non-conformal sources, on the other hand, could introduce ghostlike instabilities in the linearized theory. However, in the latter case the results of the linearized approximation are unwarranted; no conclusion on the (in)stability of the solution of the DGP model can be drawn without invoking non-linear dynamics.

An important role of non-linearities in this model have been emphasized in a somewhat different context. In a simplest case of gravity of a static source, e.g., the Sun, it is the breakdown of naive linearization that enables one to evade [25] the van Dam-Veltman-Zakharov (vDVZ) discontinuity [26, which, if present, would have ruled out the model already by the Solar system data, without any further need to consider cosmology.

Fortunately, the breakdown of the linearized perturbation theory does not necessarily mean incalculability. Instead, an expansion in other small parameters should be carried out, see, e.g., [25], 227], [28]. For instance, despite the fact that the nearby gravitational field produced by a static source (such as Sun, Earth, etc.) is incalculable within the conventional linearized approximation, the alternative approaches give rise to a weak field which approximates the result of General Relativity (GR) to a high degree of accuracy, with tiny deviations that could be potentially measurable (see [10, 11] and [13]). The fundamental reason for this is the absence of a continuous transition from DGP to GR in the linearized theory (i.e., the vDVZ discontinuity is present there), whereas, in a full non-linear model such a transition does exist, at least in exact solutions of the classical theory [25].

After these general remarks, we turn below to a brief description of the content and main results of the present work, with all the important technical details left for the bulk of the paper and Appendices.

We start in Section 2 by studying an empty self-accelerated solution, i.e., the background on which no external matter/radiation or non-linear gravitational sources are introduced. This is an artificial reduction of the theory, but it serves as an instructive playground. We look at small gravitational perturbations on this background. The linearized approximation gives a well-defined spectrum of tensor per- 
turbations, but in addition, there is a scalar mode that could or could not give rise to the ghost-like instability. We will show that for the empty brane the scalar acts as a Lagrange multiplier. Such a theory is analogous to conventional QED in which the Lorentz gauge-fixing condition is introduced by the Lagrange multiplier technique. It has been known that such a model can be quantized consistently, e.g., in the canonical approach by the Nakanishi-Lautrup method [29, 30], or more straightforwardly, by the path integral approach. Likewise, in the gravitational case at hand, we will show that the perturbations can be quantized in a path integral approach without negative norm states (i.e., without the ghost). We will discuss why our results differ from those conclusions of Ref. [23] that deal with the empty self-accelerated solution.

Things change when non-conformal matter or non-linear gravitational sources are allowed in the theory. For simplicity we consider below an external matter source $T_{\mu \nu}$. The linearized calculations suggest that $T_{\mu \nu}$ with a nonzero trace could excite a ghost-like instability ${ }^{3}$. If this were all, then any nonzero $T$ could start to radiate the ghost-like mode, rapidly destabilizing itself.

The above logic, however, misses the main feature of the DGP model, the importance of the nonlinear interactions. To put our discussions in a context let us first consider an empty brane with flat space-time as a background (this is not a self-accelerated solution yet!). Moreover, consider for simplicity a spherically symmetric matter distribution of radius $r_{0}$ and density $\rho_{0}$. The $4 \mathrm{D}$ gravitational radius of this source is $r_{M}=2 G_{N} M=2 G_{N}(4 / 3) \pi r_{0}^{3} \rho_{0}$. There is a new distance scale in the theory 25] (called the Vainshtein scale ${ }^{4}$ )

$$
r_{*} \sim\left(r_{M} / H_{0}^{2}\right)^{1 / 3}
$$

where $H_{0} \simeq 10^{-42} \mathrm{GeV}$ is the present-day value of the Hubble parameter. Below the scale (11), i.e., for $r \lesssim r_{*}$ the linearized approximation breaks down [25]. For this breakdown to be relevant, $r_{*}$ has to be bigger that $r_{0}$. This translates into the condition on the density of the source $\rho_{0} \gtrsim \rho_{c}$, where $\rho_{c} \sim 10^{-29} \mathrm{~g} / \mathrm{cm}^{3}$ is a critical density of the Universe. For such sources the invariant scalar curvature, $R$, is bigger than the space-time curvature of the observable Universe, i.e., $R \gtrsim$ $H_{0}^{2}$. Most of the sources around us satisfy this condition; we will refer to them as supercritical sources. In any realistic setup, and on the self-accelerated background in particular, the scalar curvature due to the background is already "critical" $R \sim H_{0}^{2}$; therefore, within any overdensity on top of this background, the local curvature will be supercritical $R \gtrsim H_{0}^{2}$, and one should expect the naive linearization to break down. One could certainly think of a case when one "prepares by hand" a subcritical source by distorting locally the self-accelerated background. Such a source, however, would by itself be unstable. One way for this instability to develop, is for the

\footnotetext{
${ }^{3}$ This statement, even within the linearized approach itself, needs further clarification; for details see calculations and discussions in Section 4.

${ }^{4} \mathrm{~A}$ similar scale was first found by Vainshtein in the context of $4 \mathrm{D}$ massive gravity 31 .
} 
source to reduce its size until its density becomes supercritical. This is energetically favorable since the bare mass of the supercritical sources gets screened [13: A supercritical source responds to the breakdown of the linearized approximation by creating a halo of a variable curvature which extends over a domain or size $r_{*}$. This halo screens the stress-tensor of the source. Moreover, the local curvature in the halo dominates over the curvature of the self-accelerated background itself [13].

How can nonlinear-effects change the conclusions of the linearized theory? There seem to be at least two ways, summarized in the paragraphs $[\mathrm{A}]$ and $[\mathrm{B}]$ below, and discussed in detail in Section 3.

[A] The non-perturbative halo that extends to $r_{*}$ renormalizes the stress-tensor of the source, as seen from distances larger than $r_{*}$. Hence, there is a question of matching of the linearized modes obtained for $r>r_{*}$ to "non-perturbative modes" that could potentially be calculated at $r<r_{*}$. A priori, there is no reason to believe that all the linearized solutions can be continued inside the $r_{*}$ region, and yet this is an assumption made implicitly in the works claiming the instability of the self-accelerated background. It may well be that certain perturbative solutions of the exterior region cannot be smoothly continued inside $r_{*}$. An evidence in favor of this is a solution of [13, in which the interior non-perturbative metric interpolates smoothly into a non-perturbative solution outside $r_{*}$, but not into the naive linearized solution. Although the gravitational fields are weak in both interior and exterior regions, they have non-analytic dependence on couplings/parameters of the theory and cannot be obtained in the linearized approximation.

[B] There is a known phenomenon of "linearization instability" in theories of gravity 32, see also [33, 34, 35, 36]. It is said to occur when not all the solutions of the linearized approximation can be carried over into the full nonlinear theory. Formally speaking, it arises because the nonlinear equations impose certain constraints on the results of the linearized calculations. These constraints cannot be captured in the linearized theory itself, and the linearized solutions typically fail to satisfy them. The fundamental reason why these constraints arise is this: Gauge fixing leaves the residual freedom w.r.t. the gauge transformations by the gauge functions that coincide with the Killing vectors of the corresponding background. Then, there could exist a charge, that is an integral of a Killing vector contracted with a certain current, which is a generator of these transformations. Upon quantization of the theory, the physical states should be required to be zero eigenvalue eigenstates of the charge operator (the charge is zero in the classical theory by construction). Furthermore, the requirement that this charge be zero order-by-order in the weak field expansion, imposes in the second order perturbation theory an integral constrain on the purely first order results.

The above described phenomenon is rather generic; the only condition it requires is that the charge itself be well-defined (e.g., the integral be convergent). This is certainly the case if the background space-time has a compact spatial section, then the integral in the definition of the charge is w.r.t. this compact 3-space and its convergence is guaranteed. In this case, the physical meaning of the additional 
constraint is simple - a compact 3-space cannot support an isolated "gauge charge", since its flux lines have nowhere to go.

On the self-accelerated background, similar global constraints can be found in its Euclidean continuation. In this case, the analog of a compact 3-space of the GR examples is the $S^{4}$ of the euclideanized worldvolume de Sitter (dS) space. However, there is also an additional nonlinear equation on $4 \mathrm{D}$ quantities that exists in DGP but does not arise in GR. Complementing this equation with the compactness of $S^{4}$, one finds a severe global constraint on linearized perturbations, as we will show in Section 3.

At the moment of this writing, we do not know whether the nonlinear effects discussed in paragraphs $[\mathrm{A}]$ and $[\mathrm{B}]$ improve or worsen the stability. however, the following is certain: Any claims on the (in)stability of the self-accelerated solution are unjustifiable without studying non-linearities. We emphasize these arguments here partially in response to the dissemination of an implicit (and erroneous in our opinion) assumption that the question of (in)stability in this model can be addressed just by looking at the linearized perturbations.

The right way to do calculations is different from what's usually done in GR. For a given cosmological source/overdensity, as a first step, one could calculate the perturbations in the domain $r \lesssim r_{*}$. There, it is the metric due to the source that dominates over the cosmological background. These perturbations are the most relevant ones, e.g., for the structure formation. One could use the small "graviton mass expansion" about the background of the source to see the presence of (in)stability of the theory. That the leading order results should be close to those of GR is implied by the expansion itself, however, this cannot guarantee the consistency of the results (i.e., absence of ghost and other instabilities) in the sub-leading order. These are the checks that should be done.

The above comments also concern attempts in the literature to contrast the predictions of the model with observations. In some of those cases, an assumption is being made that the values of the cosmological parameters can be extracted from the early cosmology using conventional GR, and those results could then be used to constrain the late time acceleration in DGP, see, e.g., 16. This however is not well justified: The structure formation in DGP, although is expected to be qualitatively close to that of GR [5], is nevertheless influenced by all the non-linear effects described above, and will differ from the GR results when it comes to the precision tests (see, e.g., comments in [17).

In the second part of the paper we discuss the DGP model with non-conformal sources which is artificially truncated at the linearized level. The resulting theory is ruled out already by the Solar system data, and presents only an academic interest; yet this is the model implicitly adopted in the works which ignore nonlinear effects while discussing the question of stability. We show that even in this model there are issues that have not been clarified. The main one is that of the boundary conditions and poles of the Green's functions from which the content of the physical states of the theory can be read off. This involves subtleties which we discuss in Section 4. 
We do not discuss the quantum gravity loops in the present paper. The loop corrections lead to the breakdown of the perturbation theory, like the tree level diagrams do. The scale of the breaking is rather low, and one may worry about the higher dimensional operators that can be generated at that low scale and spoil the predictivity of the theory [20, 21]. The self-accelerated solution itself is right at that breaking scale, and any perturbation about it, exceeds that scale. If the above approach is adopted, then the analysis showing that on the self-accelerated background the bending mode flips the sign of its kinetic term, and, hence, produces a ghost 21], cannot be conclusive. These issues, and ways to address them consistently, were discussed by Dvali [37, 38. In the present paper we adopt the approach $13]$ in which all the loops are calculated about some external classical background field (e.g., early cosmology, galaxies, planets etc). In this case, the loop effects are additionally suppressed by the physical scale of the background metric, and can be harmless at observable distances.

\section{Perturbations of empty self-accelerated brane}

In this Section we discuss an instructive setup with an empty self-accelerated brane. Gravity is truncated to linearized perturbations about the background that is a solution of the full nonlinear equations. There are no sources placed on such a brane; the cosmic acceleration is triggered and maintained by the gravitational sector itself, and we look at the evolution of small gravitational perturbations on such a background. The obtained results will also be applicable to the theory with conformal sources.

The DGP gravitational action takes the form [1]

$$
S=\frac{M_{\mathrm{Pl}}^{2}}{2} \int d^{4} x \sqrt{g_{4}} R\left(g_{4}\right)+\frac{M_{*}^{3}}{2} \int d^{4} x d y \sqrt{g} \mathcal{R}(g)+\ldots,
$$

where $R$ and $\mathcal{R}$ are the four-dimensional and five-dimensional Ricci scalars, respectively, and $M_{*}$ stands for the gravitational scale of the bulk theory. The analog of the graviton mass is $m_{c}=2 M_{*}^{3} / M_{\mathrm{Pl}}^{2}$. The $(4+1)$ coordinates are $x^{M}=\left(x^{\mu}, y\right)$, $\mu=0, \ldots, 3 ; g_{4}$ and $R$ are the absolute value of the determinant and $4 \mathrm{D}$ curvature for the $4 \mathrm{D}$ components $g_{4 \mu \nu}(x, y)$ of the $5 \mathrm{D}$ metric $g_{A B}(x, y)$

$$
g_{\mu \nu}(x, y=0) \equiv g_{4 \mu \nu}(x) .
$$

There is a boundary (a brane) at $y=0$ and $\mathbf{Z}_{2}$ symmetry across the boundary is imposed. The boundary Gibbons-Hawking term is implied to warrant the correct Einstein equations in the bulk. The matter fields, that are omitted in the present section, will later be assumed to be localized on a brane.

Below we consider the metric perturbations of the form:

$$
d s^{2}=\left(\bar{g}_{\mu \nu}(x, y)+\delta g_{\mu \nu}(x, y)\right) d x^{\mu} d x^{\nu}+2 \delta g_{\mu 5} d x^{\mu} d y+\left(1+\delta g_{55}(x, y)\right) d y^{2},
$$


where the background quantities will be denoted by the bar $\bar{g}_{\mu \nu}(x, y)=A^{2}(y) \gamma_{\mu \nu}(x)$. We start with the 5D equations of motion. They decompose into $\{\mu 5\},\{55\}$ and $\{\mu \nu\}$ components. Let us first look at the $\{\mu 5\},\{55\}$ equations. They read respectively

$$
\begin{array}{r}
\nabla^{\mu} K_{\mu \nu}=\nabla_{\nu} K, \\
R=K^{2}-K_{\mu \nu} K^{\mu \nu} .
\end{array}
$$

Here, $K=g^{\mu \nu} K_{\mu \nu}$ is the trace of the extrinsic curvature tensor defined as follows:

$$
K_{\mu \nu}=\frac{1}{2 N}\left(\partial_{y} g_{\mu \nu}-\nabla_{\mu} N_{\nu}-\nabla_{\nu} N_{\mu}\right)
$$

and $\nabla_{\mu}$ is a covariant derivative w.r.t. the metric $g_{\mu \nu}$. We introduced the lapse scalar field $N$, and the shift vector field $N_{\mu}$ according to the standard rules:

$$
g_{\mu 5} \equiv N_{\mu}=g_{\mu \nu} N^{\nu}, \quad g_{55} \equiv N^{2}+g_{\mu \nu} N^{\mu} N^{\nu} .
$$

Equations (5) and (6) should be satisfied everywhere in the bulk as well as on the brane. The $\{\mu \nu\}$ equation, on the other hand, takes different form depending whether it is in the bulk or on the brane

$$
\begin{aligned}
G_{\mu \nu}^{(5)} & =0 \quad \text { for } \quad y \neq 0 \\
\left.G_{\mu \nu}\right|_{y=0} & -\frac{m_{c}}{2}\left[K_{\mu \nu}-g_{\mu \nu} K\right]_{-\epsilon}^{+\epsilon}=T_{\mu \nu}(x) .
\end{aligned}
$$

\section{Spectrum of empty self-accelerated brane}

We begin by analyzing small perturbations about an empty (i.e., $T_{\mu \nu}=0$ ) selfaccelerated brane. We introduce the following self-explanatory notations

$$
\begin{gathered}
g_{\mu \nu}=\bar{g}_{\mu \nu}+\delta g_{\mu \nu}, \quad K_{\mu \nu}=\bar{K}_{\mu \nu}+\delta K_{\mu \nu} \\
\bar{K}_{\mu \nu}=n \bar{g}_{\mu \nu}, \quad \bar{K}=\bar{g}^{\mu \nu} \bar{K}_{\mu \nu}=4 n \quad n \equiv \frac{\partial_{y} A}{A}
\end{gathered}
$$

where, according to the above definitions:

$$
K=g^{\mu \nu} K_{\mu \nu} \equiv \bar{K}+\delta K, \quad \delta K=\bar{g}^{\mu \nu} \delta K_{\mu \nu}-n \bar{g}^{\mu \nu} \delta g_{\mu \nu}
$$

For the self-accelerated solution $|H|=m_{c}$. The unperturbed part of the off-diagonal equation (5) is identically satisfied. While the unperturbed parts of the $\{55\}$ and junction equations respectively give: $\bar{R}=12 \mathrm{H}^{2}$ and $\bar{R}=12 \mathrm{Hm}_{c}$; these are consistent with each other (from now on we will replace $|H|$ by $H$ with the understanding that we should always take a positive value for the latter no matter what is the direction of the time arrow). 
Let us analyze the small fluctuations described by the above system of equations. The perturbations of the off-diagonal equation (5) satisfy the following relation

$$
\nabla^{\mu}\left(\delta K_{\mu \nu}-n \delta g_{\mu \nu}\right)=\nabla_{\nu}\left(\bar{g}^{\rho \sigma} \delta K_{\rho \sigma}-n \bar{g}^{\rho \sigma} \delta g_{\rho \sigma}\right)
$$

where $\nabla^{\mu}$ is a $4 \mathrm{D}$ covariant derivative constructed out of $\bar{g}_{\mu \nu}$. We will return to this equations shortly.

For small perturbations of the $\{55\}$ equation (6) we find

$$
\delta R=6 n\left(\bar{g}^{\mu \nu} \delta K_{\mu \nu}-n \bar{g}^{\mu \nu} \delta g_{\mu \nu}\right)
$$

while from the source-free junction conditions one gets

$$
\left.\delta R\right|_{0^{+}}=\left.3 m_{c}\left(\bar{g}^{\mu \nu} \delta K_{\mu \nu}-n \bar{g}^{\mu \nu} \delta g_{\mu \nu}\right)\right|_{0+} .
$$

Since $m_{c}=H$, equations (15) and (16) are in contradiction unless the r.h.s. of (16) is zero. Moreover, requiring the continuity of the $4 \mathrm{D}$ curvature perturbations $\delta R$ we conclude that the r.h.s. of (15) should also be zero for arbitrary $y$ :

$$
\bar{g}^{\mu \nu} \delta K_{\mu \nu}-n \bar{g}^{\mu \nu} \delta g_{\mu \nu}=0 .
$$

We will come back to this relation below.

Furthermore, we need to study small perturbations of the bulk $\{\mu \nu\}$ equation. Since we are dealing with an empty brane (or the one with a conformal stress tensor), we can adopt the Gaussian normal coordinates and choose the following "gauge" 5

$$
\nabla^{\mu} \delta g_{\mu \nu}=0, \quad \bar{g}^{\mu \nu} \delta g_{\mu \nu}=0, \quad \delta g_{55}=\delta g_{\mu 5}=0
$$

In this gauge the bulk $\{\mu \nu\}$ equation takes the form:

$$
\partial_{y}^{2} \delta g_{\mu \nu}+\frac{1}{A^{2}}\left(\square_{4}-4 H^{2}\right) \delta g_{\mu \nu}=0
$$

The junction condition reads

$$
-\left.\frac{1}{2}\left(\square_{4}-4 H^{2}+H \partial_{y}\right) \delta g_{\mu \nu}\right|_{0^{+}}=0 .
$$

Let us discuss the KK modes. We use the following decomposition:

$$
\delta g_{\mu \nu}(x, y) \equiv \int h_{\mu \nu}^{(m)}(x) \tilde{f}_{m}(y) d m, \quad\left(\square_{4}-2 H^{2}\right) h_{\mu \nu}^{(m)}(x)=m^{2} h_{\mu \nu}^{(m)}(x)
$$

It is useful to turn to a new coordinate $z$ and a function $u_{m}$

$$
d z \equiv \frac{d y}{A(y)}, \quad \tilde{f}_{m} \equiv A^{1 / 2} u_{m}
$$

\footnotetext{
${ }^{5}$ In general, this is not an acceptable gauge fixing condition for the metric that couples to a non-conformal source. That is why one eventually needs to introduce the brane bending mode [40.
} 
This choice is very convenient since the bulk equation (19) and the junction conditions (20) for these variables reduce respectively to Eqs. (128) and (129) of Appendix A, where the latter equations have been solved (see also 22 ). The spectrum consists of a normalizable localized mode of mass $m_{*}^{2}=3 m_{c} H-m_{c}^{2}=2 m_{c}^{2}$ and a continuum of KK modes with masses $m_{K K}^{2} \geq 9 H^{2} / 4$. Note that because of the boundary condition that depends on the KK mass, the modes themselves are not orthogonal. The physical modes are their linear superpositions. However, this won't be important for our discussions.

Besides these states, there is a zero-mode solution, $m=0, \tilde{f}_{0}=A^{2}$ (see Appendix A for details), supported by the above system of equations. This solution is not plane-wave normalizable. Its wave-function grows in the bulk as fast as the background metric itself. When the warp-factor is scaled out, the zero-mode has a constant profile in the $y$ direction, $A^{2}(y) \gamma_{\mu \nu}(x)+A^{2}(y) h_{\mu \nu}^{\mathrm{zm}}(x)$, where $\gamma_{\mu \nu}(x)$ denotes the $4 \mathrm{D} \mathrm{dS}$ metric and $h_{\mu \nu}^{\mathrm{zm}}(x)$ is the zero-mode wave-function. We will discuss properties of this mode in Sections 4, but we ignore it for the time being.

Let us concentrate on the lowest massive localized mode mentioned above. The mass of this mode is related to the background space-time curvature as follows:

$$
m_{*}^{2}=\frac{\bar{R}}{6}=2 H^{2}
$$

It has been known for some time that 4D massive gravity on a dS background with the relation (23) exhibits an additional local symmetry [39]

$$
\delta h_{\mu \nu}^{*}=\left(\nabla_{\mu} \nabla_{\nu}+H^{2} \gamma_{\mu \nu}\right) \alpha(x)
$$

with an arbitrary $\alpha$. This enables one to gauge-remove one out of the five onshell polarizations of the massive graviton. The resulting graviton has four physical polarizations 39. Advantages and disadvantages of this theory are summarized in Appendix B. For further convenience, we will denote the Lagrangian of that theory by $\mathcal{L}_{*}$. The question that we'd like to discuss is whether the DGP Lagrangian on the self-accelerated solution reduces at low energies to $\mathcal{L}_{*}[22,23$. To answer this question we note that the local symmetry (24) is present in DGP only for the restricted gauge functions. Indeed, both bulk and brane equations of motion are invariant, in the absence of the sources (or with conformal sources only), w.r.t. the following symmetry transformations

$$
\delta g_{\mu \nu} \rightarrow \delta g_{\mu \nu}+\left(\nabla_{\mu} \nabla_{\nu}+H^{2} \gamma_{\mu \nu}\right) \sigma
$$

where the field $\sigma$ satisfies

$$
\left(\square+4 H^{2}\right) \sigma=0
$$

One can see this by performing straightforward variations of the equations presented above and using the following identities:

$$
\begin{aligned}
\left(\square-4 H^{2}\right)\left(\nabla_{\mu} \nabla_{\nu}+H^{2} \gamma_{\mu \nu}\right)(\text { scalar }) & =\left(\nabla_{\mu} \nabla_{\nu}-H^{2} \gamma_{\mu \nu}\right)\left(\square+4 H^{2}\right) \text { (scalar) } \\
\left.\nabla^{\mu}\left(\nabla_{\mu} \nabla_{\nu}+H^{2} \gamma_{\mu \nu}\right) \text { (scalar }\right) & =\nabla_{\nu}\left(\square+4 H^{2}\right) \text { (scalar) }
\end{aligned}
$$


In the absence of sources, the symmetry (25) can be used to remove on-shell a potentially dangerous degree of freedom, and fluctuations can be quantized consistently without any ghost or other pathologies, as we'll see in the next subsection.

Before we turn to these issues, however, we'd like to clarify the following point. The difference between the low energy DGP and the model $\mathcal{L}_{*}$ is not entirely clear from our discussions above. Indeed, the profile of the localized mode $u_{m_{*}}$ is known, it's 4D effective Lagrangian can be calculated by ignoring other modes and integrating the 5D action w.r.t. the $y$ coordinate. Doing so, it would naively seem, that the low-energy effective Lagrangian on the self-accelerated branch coincides with $\mathcal{L}_{*}$. However, as was shown in Ref. [23, the model, in general, does not reduce to the $\mathcal{L}_{*}$ theory. This is because of the following: In the calculation of the present section the brane bending mode was ignored, it was simply put equal to zero. This is consistent for an empty brane (more precisely, as long as $T=0$ ). As a result of this, a certain constraint, which is present in the full linearized theory, is not captured. To find this constraint, and to see whether it distinguishes DGP from $\mathcal{L}_{*}$, one should work with the bending mode manifestly present in the action, as was done in [23]. However, as we will show in the next subsection, the bending mode appears as a Lagrange multiplier. As a result, in the absence of sources, both the low-energy Lagrangian of DGP and $\mathcal{L}_{*}$ can be consistently quantized to contain four on-shell physical polarizations without ghosts or tachyons.

\section{Effective Lagrangian approach}

Let us start with an illustrative model discussed in $[23]^{6}$. The Lagrangian contains one gauge field $A_{\mu}$ and two scalars, $\varphi_{1}$ and $\varphi_{2}$ :

$$
\mathcal{L}=-\frac{1}{4} F_{\mu \nu}^{2}-\frac{f_{1}^{2}}{2}\left(\partial_{\mu} \varphi_{1}+e A_{\mu}\right)^{2}+\frac{f_{2}^{2}}{2}\left(\partial_{\mu} \varphi_{2}+e A_{\mu}\right)^{2}
$$

where $f_{1}^{2}, f_{2}^{2}$ are two positive constants. The above Lagrangian is invariant under the local symmetry transformations $\delta \varphi_{1}=\delta \varphi_{2}=\alpha, \delta A_{\mu}=-\partial_{\mu} \alpha / e$. These can be used to gauge-remove one of the two scalar fields, e.g., one can set $\varphi_{2}=0$. Following [23], consider the case

$$
f_{1}^{2}=f_{2}^{2}
$$

which presumably mimics physics of the self-accelerated solution discussed in the present paper $^{7}$. For this particular choice the Lagrangian reduces to [23]:

$$
\mathcal{L}=-\frac{1}{4} F_{\mu \nu}^{2}-\frac{f_{1}^{2}}{2}\left(\partial_{\mu} \varphi_{1}\right)^{2}-f_{1}^{2} e A^{\mu} \partial_{\mu} \varphi_{1}
$$

\footnotetext{
${ }^{6}$ The authors of [23] attribute this model to R. Rattazzi.

${ }^{7}$ The case $f_{1}^{2} \neq f_{2}^{2}$ is supposed to mimics the physics of a brane on which additional tension is introduced. This is more subtle and will not be considered in the present work.
} 
By calculating the Hamiltonian of the helicity-0 sector of the above theory (30), this model was claimed to have a ghost [23].

To look more closely into this issue, let us perform in (30) the field redefinition

$$
B_{\mu} \equiv A_{\mu}+\frac{1}{2 e} \partial_{\mu} \varphi_{1}, \quad \lambda \equiv f_{1}^{2} e \varphi_{1}
$$

In terms of the new fields the Lagrangian (30) takes the form (up to a total derivative)

$$
\mathcal{L}=-\frac{1}{4} F_{\mu \nu}^{2}+\lambda \partial^{\mu} B_{\mu}
$$

where $F_{\mu \nu}$ is a field-strength for the $B_{\mu}$ field now. This is a Lagrangian of a gauge field $B_{\mu}$ for which the Lorentz gauge-fixing condition $\partial^{\mu} B_{\mu}=0$ has been introduced by means of the Lagrange multiplier $\lambda$. It is well known how to quantize this Lagrangian consistently without the appearance of ghosts or other pathologies. For completeness we present here the summary of the path integral quantization, as well as the canonical quantization due to Nakanishi and Lautrup (NL) [29, 30].

In the path integral approach we find

$$
\int[d B][d \lambda] e^{i \int d^{4} x \mathcal{L}}=\int[d B] \delta\left(\partial^{\mu} B_{\mu}\right) e^{i \int d^{4} x\left(-\frac{1}{4} F_{\mu \nu}^{2}\right)} .
$$

The r.h.s. of the above equation is nothing but the path integral for QED with the Lorentz gauge-fixing condition where the Faddeev-Popov determinant $\operatorname{det}|\square|$, which is trivial in this case, is dropped. Needless to say that this path integral does not propagate any negative norm states (ghosts) and can be straightforwardly completed to a BRST invariant theory (the Faddeev-Popov ghost in this case are decoupled).

Counting of the on-shell degrees of freedom in the Lagrangian formalism is also straightforward; (32) imposes the Lorentz condition on the gauge field, removing one degree of freedom, yet allows for residual gauge transformations $\delta B_{\mu}=\partial_{\mu} \alpha$, where $\square \alpha=0$. This can be used to constrain on-shell the second degree of freedom, rendering two out of the four modes of $B_{\mu}$.

In the Lagrangian quantization of (32) the subsidiary Nakanishi-Lautrup condition should be imposed on physical states of the theory: $\lambda^{(-)} \mid$Phys $>=0$ (here $\lambda^{(-)}$ denotes the negative frequency part of the field $\lambda)^{8}$. The theory defined this way has no perturbative classical or quantum ghost-like instabilities [29, 30 ${ }^{9}$. Moreover,

\footnotetext{
${ }^{8}$ The NL subsidiary condition is an analog of the Gupta-Bleuler condition in the conventional Lagrangian quantization of electrodynamics.

${ }^{9}$ We point out that the Lagrangian (32), at the classical level, could have solutions that are not present in conventional electrodynamics 41. However, these solutions form different superselection sectors as they imply the presence of some additional physics (e.g., sources at infinity, different boundary conditions, etc.). We won't consider such backgrounds here since our goal is to study small perturbations on a given background (in particular, on the self-accelerated background in the gravity case discussed below).
} 
although the canonical Hamiltonian of the theory does contain negative terms, the physical Hamiltonian that acts on states of physical Hilbert space, which obey all the constraints and NL subsidiary condition, is in fact positive semi-definite.

Let us now turn to the effective Lagrangian approach to the empty self-accelerated brane. The four dimensional effective action for the localized and brane bending modes was derived in [23], which we follow below. The effective Lagrangian takes the form [23]:

$$
\mathcal{L}_{\text {eff }}=\mathcal{L}_{*}\left(A_{\mu \nu}\right)-H A^{\mu \nu}\left(\nabla_{\mu} \nabla_{\nu}-\gamma_{\mu \nu} \square-3 H^{2} \gamma_{\mu \nu}\right) \varphi-\frac{9 H^{2}}{4} \varphi\left(\square+4 H^{2}\right) \varphi,
$$

where $A_{\mu \nu} \equiv \delta g_{\mu \nu}(x, y=0)$ denotes the tensor mode and $\varphi$ is the bending mode. Furthermore, $\mathcal{L}_{*}\left(A_{\mu \nu}\right)$ denotes the Lagrangian of a linearized massive gravity on the dS background when the graviton mass and the cosmological constant satisfy a special relation $m_{*}^{2}=2 H^{2}$. The explicit expression for $\mathcal{L}_{*}\left(A_{\mu \nu}\right)$ is given in [42] (see also Appendix $\mathrm{B}$ ), but what is important here is that $\mathcal{L}_{*}\left(A_{\mu \nu}\right)$ is invariant w.r.t. the local transformation, $\delta A_{\mu \nu}=\left(\nabla_{\mu} \nabla_{\nu}+H^{2} \gamma_{\mu \nu}\right) \alpha(x)$, for an arbitrary $\alpha(x)$. As a result of this symmetry, $\mathcal{L}_{*}\left(A_{\mu \nu}\right)$ does not retain any physical scalar degrees of freedom the only on-shell modes described by $\mathcal{L}_{*}\left(A_{\mu \nu}\right)$ are 2 polarizations of a transversetraceless (TT) tensor and two polarizations of a transverse vector [39] ${ }^{10}$. However, the last two terms of $\mathcal{L}_{\text {eff }}$ are not invariant under the above local transformation. Hence, the question arises whether this reintroduces back the scalar polarization that could be a ghost.

We will show below that the results are very similar to those of a simple gaugefield example 23] considered at the beginning of this subsection. In particular, the non-invariant terms in $\mathcal{L}_{\text {eff }}$ can be recast as a product of a Lagrange multiplier with a gauge-fixing condition. Just as in the toy example, small perturbations of the resulting theory can consistently be quantized, without any ghosts. To see this, we perform a redefinition of the $A_{\mu \nu}$ field

$$
A_{\mu \nu}=B_{\mu \nu}+\left(\nabla_{\mu} \nabla_{\nu}+H^{2} \gamma_{\mu \nu}\right) \omega .
$$

This change of variables does not affect $\mathcal{L}_{*}$ since $\mathcal{L}_{*}\left(A_{\mu \nu}\right)=\mathcal{L}_{*}\left(B_{\mu \nu}\right)$. However, the last two terms of $\mathcal{L}_{\text {eff }}$ in (34) do get modified. To find the result of this let us now introduce the traceless operator $P_{\mu \nu} \equiv \nabla_{\mu} \nabla_{\nu}-\frac{1}{4} \gamma_{\mu \nu} \square$, that satisfies $P^{\mu \nu} P_{\mu \nu}($ scalar $)=\frac{3}{4} \square\left(\square+4 H^{2}\right)$ (scalar), then,

$$
\begin{aligned}
\mathcal{L}_{\text {eff }}= & \mathcal{L}_{*}\left(B_{\mu \nu}\right)-H B^{\mu \nu}\left(\nabla_{\mu} \nabla_{\nu}-\gamma_{\mu \nu} \square-3 H^{2} \gamma_{\mu \nu}\right) \varphi-\frac{9 H^{2}}{4} \varphi\left(\square+4 H^{2}\right) \varphi \\
& -H \omega\left(\nabla_{\mu} \nabla_{\nu}+H^{2} \gamma_{\mu \nu}\right)\left(P_{\mu \nu}-\frac{3}{4} \gamma_{\mu \nu}\left(\square+4 H^{2}\right)\right) \varphi \\
= & \mathcal{L}_{*}\left(B_{\mu \nu}\right)-H B^{\mu \nu}\left(\nabla_{\mu} \nabla_{\nu}-\gamma_{\mu \nu} \square-3 H^{2} \gamma_{\mu \nu}\right) \varphi-\frac{9 H^{2}}{4} \varphi\left(\square+4 H^{2}\right) \varphi \\
& +H \omega 3 H^{2}\left(\square+4 H^{2}\right) \varphi,
\end{aligned}
$$

${ }^{10}$ Notice that for $m_{*}^{2}=2 H^{2}$ there exists a unitary massive spin-two representation of the dS group with 4 degrees of freedom. 
and setting $\omega=\frac{3}{4 H} \varphi$, the effective Lagrangian becomes

$$
\mathcal{L}_{\text {eff }}=\mathcal{L}_{*}\left(B_{\mu \nu}\right)-\varphi H\left(\nabla_{\mu} \nabla_{\nu}-\gamma_{\mu \nu} \square-3 H^{2} \gamma_{\mu \nu}\right) B^{\mu \nu}
$$

Remarkably, the bending mode $\varphi$ acts as a Lagrange multiplier imposing a certain constraint $^{11}$. This is very similar in spirit to the NL form of the electrodynamics Lagrangian (32). Hence, our consideration below will be similar too. We start with the path integral quantization of this theory

$$
\int[d B][d \varphi] e^{i \int d^{4} x \mathcal{L}_{\mathrm{eff}}}=\int[d B] \delta\left(\left(\nabla_{\mu} \nabla_{\nu}-\gamma_{\mu \nu} \square-3 H^{2} \gamma_{\mu \nu}\right) B^{\mu \nu}\right) e^{i \int d^{4} x \mathcal{L}_{*}},
$$

the r.h.s. of this can be regarded as the path integral of a theory described by $\mathcal{L}_{*}$ in which the gauge fixing condition

$$
\left(\nabla_{\mu} \nabla_{\nu}-\gamma_{\mu \nu} \square-3 H^{2} \gamma_{\mu \nu}\right) B^{\mu \nu}=0
$$

is imposed. Moreover, it is straightforward to calculate that the Faddeev-Popov determinant for the gauge condition (39) takes a form, $\operatorname{det}\left|\square+4 H^{2}\right|$. This is a trivial factor (independent of $B$ ) which can be restored in the r.h.s. of (38) at the expense of an arbitrary overall prefactor. The result reads

$$
\int[d B] \delta\left(\left(\nabla_{\mu} \nabla_{\nu}-\gamma_{\mu \nu} \square-3 H^{2} \gamma_{\mu \nu}\right) B^{\mu \nu}\right) \operatorname{det}\left|\square+4 H^{2}\right| e^{i \int d^{4} x \mathcal{L}_{*}} .
$$

This is nothing but the path integral for the theory $\mathcal{L}_{*}$ with the gauge fixing and Faddeev-Popov determinant terms. Therefore, we conclude that the quantization of $\mathcal{L}_{\text {eff }}$ is identical to the quantization of $\mathcal{L}_{*}$ and both of these theories (in the absence of sources) propagate 4 physical on-shell polarizations and no ghosts.

Let us also count degrees of freedom in the Lagrangian formalism. We first recall how things work in the theory described by $\mathcal{L}_{*}$. The equations of motion and Bianchi identities lead to the condition $\nabla^{\mu} B_{\mu \nu}=\nabla_{\nu} B$ (see Appendix B). This equation, as well as the Lagrangian $\mathcal{L}_{*}$ itself, are invariant under the local transformations $\delta B_{\mu \nu}=\left(\nabla_{\mu} \nabla_{\nu}+H^{2} \gamma_{\mu \nu}\right) \alpha(x)$, with an arbitrary $\alpha$. This can be used to gauge remove the trace, i.e., set $B_{\mu}^{\mu}=0$. As a result, we also get $\nabla^{\mu} B_{\mu \nu}=0$. Hence, the $B_{\mu \nu}$ field is transverse-traceless, hence, it has only 5 independent components. However, there still remains gauge symmetry under the transformations $\delta B_{\mu \nu}=$ $\left(\nabla_{\mu} \nabla_{\nu}+H^{2} \gamma_{\mu \nu}\right) \sigma(x)$, where $\left(\square+4 H^{2}\right) \sigma=0$. This allows to eliminate one more on-shell degree of freedom. Hence, $B_{\mu \nu}$ field in the Lagrangian $\mathcal{L}_{*}$ describes only 4 physical polarizations [39 ${ }^{12}$.

Let us now see how the counting works for $\mathcal{L}_{\text {eff }}$. Variation w.r.t. $\varphi$ yields (39). Furthermore, it is straightforward to check that the Bianchi identities for the $\{\mu \nu\}$

\footnotetext{
${ }^{11}$ Notice that $\left(\nabla_{\mu} \nabla_{\nu}-\gamma_{\mu \nu} \square-3 H^{2} \gamma_{\mu \nu}\right) B^{\mu \nu} \sim \delta R$.

${ }^{12}$ The Lagrangian $\mathcal{L}_{*}$, however, does not permit the $B_{\mu \nu}$ field to be coupled to a non-conformal source, $T \neq 0$ (see e.g., Appendix B). This is very similar to how the conventional electrodynamics does not permit the photon to be coupled to a non-conserved current.
} 
equation lead to $\nabla^{\mu} B_{\mu \nu}=\nabla_{\nu} B$. The divergence of the latter combined with (39) leads to $B=0$, and, as a consequence, $\nabla^{\mu} B_{\mu \nu}=0$ too. Moreover, the Lagrangian $\mathcal{L}_{\text {eff }}$, as well as all the above constraints, are still invariant under the transformations $\delta B_{\mu \nu}=\left(\nabla_{\mu} \nabla_{\nu}+H^{2} \gamma_{\mu \nu}\right) \sigma(x)$, where $\left(\square+4 H^{2}\right) \sigma=0$. These can be used to eliminate one more degree of freedom of the $B_{\mu \nu}$ field, reducing the total number of its physical on-shell states down to 4 . Therefore, as far as the $B_{\mu \nu}$ field is concerned, there is no difference between the description in terms of $\mathcal{L}_{*}$ or $\mathcal{L}_{\text {eff }}$. Let us now look at the $\varphi$ field. This field is a Lagrange multiplier in (37). Moreover, the trace of the $\{\mu \nu\}$ equation plus the Bianchi identities lead to $\left(\square+4 H^{2}\right) \varphi=0$. This is reminiscent of the condition $\square \lambda=0$ on the Lagrange multiplier emerging in (32). As in that case, the value of the Lagrange multiplier $\varphi$ can be set to zero and this condition should be possible to maintained in the full quantum theory by imposing the NL like constraint on the physical states $\varphi^{(-)} \mid$Phys $\rangle=0$. This must be sufficient to guarantee that the small perturbations described by the Lagrangian $\mathcal{L}_{\text {eff }}(B)$ yield no ghosts, as it was shown in the path integral quantization. Moreover, the physical degrees of freedom are identical to those described by $\mathcal{L}_{*}$.

Summarizing, we showed that small perturbations on the empty self-accelerated brane can be quantized consistently and do not exhibit any ghost instability. These results also apply to the brane endowed with conformal sources. This is because all the arguments and counting of the degrees of freedom go through in the presence of conformal sources, since the symmetry (25), (26) is preserved in that case ${ }^{13}$.

The above presented counting of the degrees of freedom in $\mathcal{L}_{\text {eff }}$ changes once sources with $T \neq 0$ are introduced. Details are given in Section 4 ; here we just note that although the relation $\nabla^{\mu} B_{\mu \nu}=\nabla_{\nu} B$ remains intact, one looses the symmetry (25), (26) that was essential to remove one on-shell degree of freedom. The physical metric that couples to the stress-tensor is

$$
\delta \tilde{g}_{\mu \nu}=\delta g_{\mu \nu}+\frac{2 A(y)}{H}\left(\nabla_{\mu} \nabla_{\nu}+H^{2} \gamma_{\mu \nu}\right) \varphi(x)
$$

Moreover, $\left(\square+4 H^{2}\right) \varphi \sim T$, and a nonzero-trace source could excite a ghost and become unstable. However, such a source also leads to the breakdown of the linearized approach adopted above. We turn to the discussions of these issues in Section 3.

\section{Perturbations in the presence of sources}

As we have already discussed in Sections 1 and 2, non-conformal sources could excite ghost in the linearized reduction of the theory (see, however, discussions in Section 4). If this were all, the sources would start to radiate ghosts until they'd disappear

\footnotetext{
${ }^{13}$ In this paper we do not discuss stability of the background w.r.t. possible classical, and semiclassical non-perturbative effects, such as the decay of the vacuum etc. If these instabilities are present, we'd expect them to set in very slowly, without affecting the observations; moreover, the full nonlinear theory should be used to study their consequences.
} 
(if the sources carry no conserved charges), or would accrete some negative "gradient energy" to produce an effective source that by itself is conformal. However, in all these considerations it is important to remember that non-conformal sources lead to the breakdown of the linearized approximation; they surround themselves with a nonlinear halo of a variable curvature that locally dominates over the self-accelerated background. The naive linearized solutions, that could contain instabilities, are not guaranteed to penetrate the halo. We will present an evidence that the linearized solutions are indeed spurious in this case. Dynamically, the halo screens the bare mass of the source, and because of this, the process of its formation is energetically favorable.

\section{Complete set of nonlinear equations}

To discuss these issues it is useful to start with the DGP action in the ADM formalism [43] (see, e.g., 44], 45]):

$$
S=\frac{M_{\mathrm{Pl}}^{2}}{2} \int d^{4} x d y \sqrt{g}\left(R \delta(y)+\frac{m_{c}}{2} N\left(R+K^{2}-K_{\mu \nu} K^{\mu \nu}\right)\right) .
$$

Equations of motion of the theory are obtained by varying the action (42) w.r.t. $g_{\mu \nu}, N$ and $N_{\mu}$. Here we reiterate a subset of two equations, the junction condition across the brane, and the $\{55\}$ equation that can be obtained by varying the action w.r.t. $N$. The former reads as follows:

$$
G_{\mu \nu}-m_{c}\left(K_{\mu \nu}-g_{\mu \nu} K\right)=T_{\mu \nu} / M_{\mathrm{Pl}}^{2}
$$

where $G_{\mu \nu}$ is the $4 \mathrm{D}$ Einstein tensor of the induced metric $g_{\mu \nu}$ and $T_{\mu \nu}$ is the matter stress tensor. The $\{55\}$ equation takes the form

$$
R=K^{2}-K_{\mu \nu}^{2}
$$

Note that (43) is valid only at $y=0^{+}$while (44) should be fulfilled for arbitrary $y$. The terms with the extrinsic curvature contain derivatives w.r.t. the extra coordinate as well as the $\{55\}$ and $\{\mu 5\}$ components of the metric. By expressing from (43) $K$ and $K_{\mu \nu}$ in terms of $R_{\mu \nu}$ and $T_{\mu \nu}$ we obtain the following expression:

$$
m_{c}^{2} R=\frac{1}{3}\left(R^{2}-3 R_{\mu \nu}^{2}\right)+\frac{1}{M_{\mathrm{Pl}}^{2}}\left(2 R_{\mu \nu} T^{\mu \nu}-\frac{1}{3} R T\right)-\frac{1}{M_{\mathrm{Pl}}^{4}}\left(T_{\mu \nu}^{2}-\frac{1}{3} T^{2}\right)
$$

The remaining equations correspond to the $\{\mu 5\}$ and bulk $\{\mu \nu\}$ equations. The $\{\mu 5\}$ equation for arbitrary $y$ (which we copy here again for convenience) reads as follows:

$$
\nabla_{\mu} K=\nabla^{\nu} K_{\mu \nu}
$$

The covariant derivative in the above equation is the one for $g_{\mu \nu}$. At $y=0$ Eq. (46) is trivially satisfied due to (43). For $y \neq 0$, (46) sets the relation between $N_{\mu}, N$ 
and $g_{\mu \nu}$. Hence, (46) gives a relation between these quantities for both $y=0$ and $y \neq 0$.

One can use the bulk $\{55\}$ equation (44) to determine $N$ in terms of $N_{\mu}$ and $g_{\mu \nu}$, and then use (46) in order to express $N_{\mu}$ in terms of $g_{\mu \nu}$. If so, there must exist one more equation which should allow to determine the bulk $g_{\mu \nu}$ itself. This is the bulk $\{\mu \nu\}$ equation, to which we turn now. The latter can be written in a few different ways. For the case at hand, the formalism by Shiromizu, Maeda and Sasaki [46] is most convenient. In this approach, the bulk $\{\mu \nu\}$ equation and the junction condition can be combined to yield a $\{\mu \nu\}$ equation at $y \rightarrow 0^{+}$. This gives a "projection" of the bulk $\{\mu \nu\}$ equation onto the brane. Since the bulk itself is empty in our case, this is the most restrictive form one can work with. The equation reads as follows [46, 47, 48, 49]:

$$
G_{\mu \nu}=\frac{1}{m_{c}^{2}} B_{\mu \nu}-E_{\mu \nu}
$$

where

$$
\begin{aligned}
B_{\mu \nu} & \equiv-\tilde{G}_{\mu \alpha} \tilde{G}_{\nu}^{\alpha}+\frac{1}{3} \tilde{G}_{\alpha}^{\alpha} \tilde{G}_{\mu \nu}+\frac{1}{2} g_{\mu \nu} \tilde{G}_{\alpha \beta} \tilde{G}^{\alpha \beta}-\frac{1}{6} g_{\mu \nu}\left(\tilde{G}_{\alpha}^{\alpha}\right)^{2} \\
\tilde{G}_{\mu \nu} & \equiv G_{\mu \nu}-T_{\mu \nu} / M_{\mathrm{Pl}}^{2}
\end{aligned}
$$

and where all quantities are taken at $y=0^{+} . G_{\mu \nu}$ denotes the 4D Einstein tensor of the metric $g_{\mu \nu}, E_{\mu \nu}$ denotes the electric part of the bulk Weyl tensor, projected onto the brane. An important property of the Weyl tensor is that it is invariant under conformal transformations. Moreover, $E_{\mu \nu}$ is traceless.

\section{Perturbations in nonperturbative domain}

One way in which the presence of instability was argued in the literature [21] is by looking at the scalar bending mode and observing that its kinetic term flips the sign due to curvature of the self-accelerated background. However, the scalar mode carries no gauge-independent physical information ${ }^{14}$. Because of this, no conclusion can be obtained just by looking at this mode; as we have seen in Section 2, the mixing with the tensor should be taken into account.

To emphasize once again how misleading the scalar mode could be, we briefly recall below a well-known example. Consider conventional 4D GR coupled to matter on a flat space. No instabilities are present in this theory. Let us perform now conformal transformation of the metric by the conformal factor $(1+\pi)$. The resulting Lagrangian contains the tensor field, kinetic mixing term of the tensor to $\pi$, and, most importantly, a wrong-sign (ghost-like) kinetic term for the scalar $\pi$. Moreover, this scalar mode does couple to matter in the new frame. If one simply ignores

\footnotetext{
${ }^{14}$ This is true even in the so called decoupling limit where as was shown in 14, the scalar mode does not decouple in DGP from the tensor modes.
} 
the tensor mode (by putting it equal to zero, or by considering fields on which the conformally rescaled $R$ is zero), one would naively conclude that $\pi$ is a ghost that could be emitted by the source. However, this would be an erroneous conclusion, since we started with the theory with no ghost in the first place. The error is made by neglecting the mixing of $\pi$ with the tensor, and ignoring the fact that $\pi$ by itself carries no physical information.

The above example is instructive, and does certainly demonstrate the danger of drawing conclusions in gravity (as well as in gauge theories) based on the helicity-0 sector alone. However, the situation is not as simple in the DGP model. This is because there are nonlinear mixing terms between the scalar and tensor modes which, on classical backgrounds, give rise to quadratic mixing terms between them. Because of this, as we will argue below, what determines the mixing at scales $r \lesssim r_{*}$, is the local background due to the source, and not the global cosmological background ${ }^{15}$.

Let us consider small perturbations around a classical solution (e.g., such as the nonperturbative solutions of [13) where there is significant curvature for distances smaller than a certain distance $r_{*}$. Taking perturbations of the following form:

$$
R_{\mu \nu}=R_{\mu \nu}^{\mathrm{cl}}+\delta R_{\mu \nu}
$$

we obtain from the first order expansion of (45)

$$
m_{c}^{2} \delta R=\frac{2}{3} R^{\mathrm{cl}} \delta R-2 R_{\mu \nu}^{\mathrm{cl}} \delta R^{\mu \nu}+\frac{2}{M_{\mathrm{Pl}}^{2}} R_{\mu \nu}^{\mathrm{cl}} T^{\mu \nu}-\frac{1}{3 M_{\mathrm{Pl}}^{2}} R^{\mathrm{cl}} T .
$$

Notice the crucial role played by the non-vanishing curvature of the classical solutions in determining the properties of the perturbations.

Let us first discuss the conventional branch. In this case, for distances much greater than $r_{*}$ the Ricci tensor is negligible. This yields $\delta R=0$ (we consider 4D sources localized to a region smaller than $r_{*}$ ). While in the regime of distances smaller than $r_{*}$ we get

$$
m_{c}^{2} \delta R=\frac{2}{3} R^{\mathrm{sch}} \delta R-2 R_{\mu \nu}^{\mathrm{sch}} \delta R^{\mu \nu}+\frac{2}{M_{\mathrm{Pl}}^{2}} R_{\mu \nu}^{\mathrm{sch}} T^{\mu \nu}-\frac{1}{3 M_{\mathrm{Pl}}^{2}} R^{\mathrm{sch}} T,
$$

where the superscript "sch" refers to the nonzero curvature solution in the conventional branch.

On the self-accelerated branch, in the large distance regime where the curvature is de Sitter like (with Hubble parameter equal to $m_{c}$ ) Eq. (50) reduces to $\delta R=-2 \delta R$,

\footnotetext{
${ }^{15}$ It is worth mentioning that the dynamics of small perturbations of the bending mode $\pi$ of Refs. 20. 21, in the nonperturbative regime, is completely dominated by the local background. That is to say, the fluctuations of the $\pi$ field are well above the strong coupling scale of the theory, the later being determined via the graviton scattering amplitude on Minkowski background 20. However, the "effective" strong coupling scale of the theory, even in the case of the graviton scattering on an empty space, is determined by the local non-perturbative background due to gravitons themselves, or due to any other supercritical sources which are present in a given setup. This effective scale is much higher than the strong coupling scale of [20] (see, discussions in the first reference of [13]).
} 
which amounts to $\delta R=0$ as in the conventional branch. In the short distance regime the curvature flips the sign with respect to the conventional branch ${ }^{16}$ :

$$
R_{\mu \nu}^{\mathrm{sch}} \rightarrow-R_{\mu \nu}^{\mathrm{sch}}
$$

Therefore, (50) yields, in this case

$$
m_{c}^{2} \delta R=-\frac{2}{3} R^{\mathrm{sch}} \delta R+2 R_{\mu \nu}^{\mathrm{sch}} \delta R^{\mu \nu}-\frac{2}{M_{\mathrm{Pl}}^{2}} R_{\mu \nu}^{\mathrm{sch}} T^{\mu \nu}+\frac{1}{3 M_{\mathrm{Pl}}^{2}} R^{\mathrm{sch}} T
$$

with only the sign differences of the r.h.s. as compared to (51).

At scales below $r_{*}$ the local curvature $R^{\text {sch }}$ overcomes $m_{c}^{2}$, and becomes larger and larger as one approaches the source (still remaining much smaller that the magnitude of the Riemann tensor components of the conventional 4D Schwarzschild metric [13]). Because of this, the expressions on the l.h.s. of (51) and (153) can be neglected as compared to the terms on the r.h.s. In this approximation, the above two equations coincide up to an overall sign.

Very similar calculations can be performed with a more general equation (47). Since the conclusions of those studies are analogous to what we found above, and, moreover, the above presented expressions can be obtained by taking trace of (47), we won't present those results here.

Therefore, the dynamics of small perturbations at scales $r \lesssim r_{*}$ is completely determined by the local metric due to the source, and not by the cosmological background. The linearized perturbations, which by themselves are valid at $r \gtrsim r_{*}$, are not guaranteed to match upon the solutions inside the $r \lesssim r_{*}$ domain. Physically, this is because the halo of a variable curvature that extends to scales $r \sim r_{*}$, screens the stress-tensor of the source [13, making it hard for the naive linearized solutions to match it smoothly. For instance, in the static solution of 13, the modes that at $r \gtrsim r_{*}$ match smoothly the interior solution, are not the modes of the naive linearized approximation. The latter are spurious.

\section{Global constraints on self-accelerated background}

In this subsection we consider Euclidean continuation of the self-accelerated background and discuss the global constraints imposed on the linearized perturbations in a theory with, and without, sources.

We start with the constraints similar to the ones emerging in $4 \mathrm{D}$ GR on a dS space-time with a closed spatial section. This part of our presentation is a straightforward generalization to one more dimension of the approach of Ref. [35]. Later in this subsection we will discuss the constraints that are more specific to the DGP model. However, we should point out that, in terms of the Lorentzian signature space-time, the results of the present section are specific to a foliation of the dS

\footnotetext{
${ }^{16}$ This statement is a direct consequence of the structure of the Israel junction conditions in DGP, and is independent of particular details of the solutions at hand.
} 
space-time that covers the whole $\mathrm{dS}$ hyperboloid. We do not expect similar results to arise for flat and open dS backgrounds in the absence of non-conformal sources. On the other hand, if the non-conformal sources were present, such global constraints could formally arise for different reasons. However, since in the latter case the linearization breaks down, these constraints should have no importance for the self-accelerated solution.

Consider the euclideanized dS space, $S^{4}$. Suppose there exists some symmetric tensor $\pi^{\mu \nu}$ which is covariantly conserved

$$
\nabla_{\nu} \pi^{\mu \nu}=0
$$

with $\nabla_{\nu}$ being the covariant derivative compatible with the metric $g_{\mu \nu}$. Then, the integral below is a total derivative, and, assuming that fields are single-valued, it vanishes on the $S^{4}$

$$
Q_{\zeta} \equiv \int_{S^{4}} d^{4} x \sqrt{g}\left(£_{\zeta} g_{\mu \nu}\right) \pi^{\mu \nu}=0
$$

The Lie derivative $£_{\zeta}$ w.r.t. the vector field $\zeta^{\alpha}$ is defined as follows

$$
\begin{aligned}
£_{\zeta} t_{\mu \nu} & =\zeta^{\alpha} \partial_{\alpha} t_{\mu \nu}+t_{\mu \alpha} \partial_{\nu} \zeta^{\alpha}+t_{\nu \alpha} \partial_{\mu} \zeta^{\alpha} \\
& =\zeta^{\alpha} \tilde{\nabla}_{\alpha} t_{\mu \nu}+t_{\mu \alpha} \tilde{\nabla}_{\nu} \zeta^{\alpha}+t_{\nu \alpha} \tilde{\nabla}_{\mu} \zeta^{\alpha}
\end{aligned}
$$

where $t_{\mu \nu}$ is an arbitrary smooth tensor and the second line follows from the first one given that $\tilde{\nabla}_{\nu}$ is a covariant derivative of an arbitrary metric whose affine connection is torsion-free ( $g_{\mu \nu}$ is one example, but not the only one).

Let us split $g_{\mu \nu}$ and $\pi^{\mu \nu}$ in their background and perturbative parts

$$
g_{\mu \nu} \equiv \gamma_{\mu \nu}+h_{\mu \nu}, \quad \pi^{\mu \nu}=\hat{\pi}^{\mu \nu}+p^{\mu \nu}
$$

Suppose that $\zeta^{\alpha}$ is a Killing vector of the background space-time, that is

$$
£_{\zeta} \gamma_{\mu \nu}=0
$$

Moreover, below we will consider $\pi^{\mu \nu}$ 's for which

$$
£_{\zeta} \hat{\pi}^{\mu \nu}=0 .
$$

Using these properties we expand the expression for $Q_{\zeta}$ (55) up to the second order in small perturbations. Ignoring the surface terms on the $S^{4}$, we get

$$
Q_{\zeta} \simeq \int d^{4} x \sqrt{\gamma}\left(£_{\zeta} h_{\mu \nu}^{(1)}\right) p^{(1) \mu \nu}=0
$$

Here $h^{(1)}$ and $p^{(1)}$ are the solutions of the first order equations. Expression (60) is an additional constraint imposed on these perturbations. 
Let us now turn to the expression for $\pi^{\mu \nu}$ that are specific to the model at hand. There are at least three tensors that satisfy the properties of $\pi^{\mu \nu}$ described above. These are:

$$
K^{\mu \nu}-g^{\mu \nu} K, \quad G^{\mu \nu}, \quad T^{\mu \nu} .
$$

Only two of these three are independent as they are related to each other by the junction condition $G^{\mu \nu}-m_{c}\left(K^{\mu \nu}-g^{\mu \nu} K\right)=T^{\mu \nu} / M_{\mathrm{Pl}}^{2}$. The global constraint (60) for these tensors reads respectfully:

$$
\begin{aligned}
Q_{\zeta}^{K} & =\int d^{4} x \sqrt{\gamma}\left(£_{\zeta} h_{\mu \nu}^{(1)}\right)\left(K^{(1) \mu \nu}-\left(g^{\mu \nu} K\right)^{(1)}\right)=0, \\
Q_{\zeta}^{G} & =\int d^{4} x \sqrt{\gamma}\left(£_{\zeta} h_{\mu \nu}^{(1)}\right) G^{(1) \mu \nu}=0, \\
Q_{\zeta}^{T} & =\int d^{4} x \sqrt{\gamma}\left(£_{\zeta} h_{\mu \nu}^{(1)}\right) T^{(1) \mu \nu}=0 .
\end{aligned}
$$

Any one of the above constraints follows from the other two. Let us focus on the last one (64). In an expanded form it reads:

$$
\int d^{4} x \sqrt{\gamma}\left(\zeta^{\alpha} \nabla_{\alpha}^{(\gamma)} h_{\mu \nu}^{(1)}+h_{\mu \alpha}^{(1)} \nabla_{\nu}^{(\gamma)} \zeta^{\alpha}+h_{\nu \alpha}^{(1)} \nabla_{\mu}^{(\gamma)} \zeta^{\alpha}\right) T^{(1) \mu \nu}=0
$$

where $\nabla_{\alpha}^{(\gamma)}$ denotes the covariant derivative compatible with the background metric $\gamma_{\mu \nu}$. The significance of this constraint is the following. one would calculate $h^{(1)}$ in terms of the $\nabla_{\alpha}^{(\gamma)}$ and an arbitrary source $T^{(1)}$ (as it is done in the next section). The question whether the obtained $h^{(1)}$ can or cannot be continued into the full nonlinear theory would have been decided based on whether or not it could satisfy the constraint (65). The latter, in general, is hard to be fulfilled automatically, and it would impose a constraint on the acceptable sources $T^{(1)}$. However, in the case of the self-accelerated solution with the sources the situation is slightly different. Local curvature within an arbitrary overdensity above the background is bigger than $m_{c}^{2}$. As a result, the perturbative expansion should break down for all these sources. Because of this, the derivation of (65) presented above fails. The effects of the nonperturbative domain lead to screening of the mass of the source 14.

In the remainder of this subsection, we would like to discuss the global constraints on perturbations of the self-accelerated background without sources. These are more specific to the DGP model, as they are based on a nonlinear trace equation that is very different from that of GR. However, as we mentioned at the beginning of this subsection, these constraints are specific to the foliation of the dS space-time that covers the whole dS hyperboloid.

To get to the point as quickly as possible we again turn to the euclideanized version of the background dS space with the topology of a four-sphere $S^{4}$, and look at one of the exact equations of motion of the DGP model

$$
3 m_{c}^{2} R=R^{2}-3 R_{\mu \nu}^{2}
$$


This equation is valid at the position of the brane in an arbitrary coordinate system. We will be expanding this equation about the background $S^{4}$ up to the second order in perturbations (which corresponds to the expansion up to the cubic order in the action). Hence,

$$
g_{\mu \nu}=\gamma_{\mu \nu}+\delta g_{\mu \nu}, \quad g_{\mu \nu}=\gamma^{\mu \nu}-\delta g^{\mu \nu}+\delta g^{\mu \alpha} \delta g_{\alpha}^{\nu}+\ldots
$$

where the first equation in (67) is just the definition of $\delta g_{\mu \nu}$. Furthermore, we use the following standard notations for the expansion of the Ricci tensor:

$$
R_{\mu \nu}=3 H^{2} \gamma_{\mu \nu}+R_{\mu \nu}^{(1)}+R_{\mu \nu}^{(2)}
$$

where $R_{\mu \nu}^{(1)}$ and $R_{\mu \nu}^{(2)}$ denote respectively the linear and quadratic in perturbations terms (for convenience, the explicit expressions for these quantities are given in Appendix C, see also [50]). It is also convenient to adopt the following notations:

$$
\delta g_{\mu \nu}=h_{\mu \nu}^{(1)}+h_{\mu \nu}^{(2)}+\ldots
$$

where $h_{\mu \nu}^{(1)}$ denotes a solution of the first order equation, while $h_{\mu \nu}^{(2)}$ is a solution of the second order equations. The decomposition of (69) is useful for keeping track of terms in the corresponding orders. For instance, the term $R_{\mu \nu}^{(1)}\left(h^{(1)}\right)$ is a first order quantity, while the terms $R_{\mu \nu}^{(1)}\left(h^{(2)}\right), R_{\mu \nu}^{(2)}\left(h^{(1)}\right)$ and $h^{(1) \mu \nu} R_{\mu \nu}^{(1)}\left(h^{(1)}\right)$ are all the second order quantities. On the other hand, the terms $h^{(2) \mu \nu} R_{\mu \nu}^{(1)}\left(h^{(1)}\right), h^{(1) \mu \nu} R_{\mu \nu}^{(2)}\left(h^{(1)}\right)$ appear in the third order, while $R_{\mu \nu}^{(2)}\left(h^{(2)}\right)$ in the fourth order. We will be ignoring all the terms beyond the second order.

To proceed further we need to specify the gauge conditions at each order. In the first order we choose the gauge

$$
\gamma^{\mu \nu} h_{\mu \nu}^{(1)}=0
$$

The first order source free equations of motion also allow to set (although the latter is not necessary for our derivations)

$$
\nabla^{\mu} h_{\mu \nu}^{(1)}=0
$$

In the second order, however, we impose only the tracelessness condition ${ }^{17}$ :

$$
\gamma^{\mu \nu} h_{\mu \nu}^{(2)}=0 .
$$

\footnotetext{
${ }^{17}$ This does not fix the gauge freedom in the second order completely, but is enough for our purposes, i.e., our considerations apply irrespective of an additional gauge fixing condition that one may need to introduce to fix the residual gauge freedom of the second order perturbations, as long as that conditions is consistent itself. Notice that we do not impose the covariant transversality condition in the second order, as this would have over-constrained the system.
} 
Now we are ready to proceed to the expansion of (66). Since $m_{c}=H$ and $\gamma^{\mu \nu} R_{\mu \nu}^{(1)}\left(h^{(1)}\right)=$ 0 , we find in the second order in perturbations:

$$
\begin{array}{r}
R \equiv g^{\mu \nu} R_{\mu \nu}=12 H^{2}+\gamma^{\mu \nu} R_{\mu \nu}^{(1)}\left(h^{(2)}\right)+\gamma^{\mu \nu} R_{\mu \nu}^{(2)}\left(h^{(1)}\right) \\
-h^{(1) \mu \nu} R_{\mu \nu}^{(1)}\left(h^{(1)}\right)+3 H^{2}\left(h_{\mu \nu}^{(1)}\right)^{2} .
\end{array}
$$

The terms on the r.h.s. of (66) are expanded to the second order as follows:

$$
\begin{array}{r}
R^{2}=144 H^{4}+24 H^{2} \gamma^{\mu \nu} R_{\mu \nu}^{(1)}\left(h^{(2)}\right)+24 H^{2} \gamma^{\mu \nu} R_{\mu \nu}^{(2)}\left(h^{(1)}\right) \\
-24 H^{2} h^{(1) \mu \nu} R_{\mu \nu}^{(1)}\left(h^{(1)}\right)+72 H^{4}\left(h_{\mu \nu}^{(1)}\right)^{2},
\end{array}
$$

and

$$
\begin{array}{r}
R_{\mu \nu}^{2} \equiv R_{\mu \nu} R_{\alpha \beta} g^{\mu \alpha} g^{\nu \beta}=36 H^{4}+6 H^{2} \gamma^{\mu \nu} R_{\mu \nu}^{(1)}\left(h^{(2)}\right)+6 H^{2} \gamma^{\mu \nu} R_{\mu \nu}^{(2)}\left(h^{(1)}\right) \\
-12 H^{2} h^{(1) \mu \nu} R_{\mu \nu}^{(1)}\left(h^{(1)}\right)+27 H^{4}\left(h_{\mu \nu}^{(1)}\right)^{2}+\left(R_{\mu \nu}^{(1)}\left(h^{(1)}\right)\right)^{2} .
\end{array}
$$

Substituting the expressions (173), (74) and (75) into the equation (66) we get

$$
\begin{array}{r}
3 H^{2} \gamma^{\mu \nu} R_{\mu \nu}^{(1)}\left(h^{(2)}\right)+3 H^{2} \gamma^{\mu \nu} R_{\mu \nu}^{(2)}\left(h^{(1)}\right)+15 H^{2} h^{(1) \mu \nu} R_{\mu \nu}^{(1)}\left(h^{(1)}\right) \\
-18 H^{4}\left(h_{\mu \nu}^{(1)}\right)^{2}-3\left(R_{\mu \nu}^{(1)}\left(h^{(1)}\right)\right)^{2}=0 .
\end{array}
$$

All the terms in the above equation are of the second order. This equation should be used to determine the second order metric perturbation $h_{\mu \nu}^{(2)}$ in terms of the squares of the first order perturbation $h_{\mu \nu}^{(1)}$ and its derivatives. However, there is more to it: Only the first term on the 1.h.s. of (76) contains the second order metric perturbation $h_{\mu \nu}^{(2)}$, the rest contains only the first order metric $h_{\mu \nu}^{(1)}$. Moreover, since $h_{\mu \nu}^{(2)}$ is traceless, then $\gamma^{\mu \nu} R_{\mu \nu}^{(1)}\left(h^{(2)}\right)=\nabla^{\mu} \nabla^{\nu} h_{\mu \nu}^{(2)}$. As a result, the first term on the l.h.s. of (76) , which also happens to be the only term containing $h_{\mu \nu}^{(2)}$, is a total derivative. Therefore, if we integrate both sides of (176) w.r.t. the background space, which in our case in $S^{4}$, the term containing $h_{\mu \nu}^{(2)}$ will drop out as a total derivative. The resulting integral equations reads:

$$
\int_{S^{4}} d^{4} x \sqrt{\gamma}\left(H^{2} \gamma^{\mu \nu} R_{\mu \nu}^{(2)}\left(h^{(1)}\right)+5 H^{2} h^{(1) \mu \nu} R_{\mu \nu}^{(1)}\left(h^{(1)}\right)-6 H^{4}\left(h_{\mu \nu}^{(1)}\right)^{2}-\left(R_{\mu \nu}^{(1)}\left(h^{(1)}\right)\right)^{2}\right)
$$

This equation contains only the first order perturbations $h_{\mu \nu}^{(1)}$, and represents an additional constraint on these perturbations imposed by the second order equations. This constraint cannot be captured in the linearized theory. Although we derived (176) using a particular gauge, it represents an integral of a gauge-invariant equation (66) $^{18}$.

\footnotetext{
${ }^{18}$ In conventional GR on $S^{4} \mathrm{Eq}$. (73) can be used to derive a global constraint.
} 
Can the first order perturbations that are solutions of the linearized equations satisfy the above constraint? To answer this question we use the explicit expressions for the quantities in (77) (see, e.g., Appendix C), which after integration by parts yields:

$$
\int_{S^{4}} d^{4} x \sqrt{\gamma}\left(\left(\square h_{\mu \nu}^{(1)}\right)^{2}+5 H^{2}\left(\nabla_{\alpha} h_{\mu \nu}^{(1)}\right)^{2}\right)=0 .
$$

The integrand on the l.h.s. of (78) is a positive semi-definite quantity in the Euclidean space that we are dealing with. Hence, the global constraint (78) implies that $\square h_{\mu \nu}^{(1)}=0$, and $\nabla_{\alpha} h_{\mu \nu}^{(1)}=0$. These can only be satisfied by trivial solutions!

Summarizing, the nonlinear equations impose very severe constraints on euclideanized linear perturbations. If fact, on a brane with no matter sources considered above, no non-trivial perturbations appear to survive these constraints. Then what is physics of perturbations on such a background? The answer to this question, as we discussed before, lies in non-perturbative dynamics. It so happens that realistic sources, whether they are due to nonlinear self-interactions of gravity itself, or external matter, give rise to the breakdown of the naive linearized approximation used in this section. This breakdown obfuscates the meaning of the conventional linearization approximation and constraints discussed above. On the other hand, it also suggests that the required calculations should be done using different approximations, e.g., the one we briefly discussed in Section 1.

\section{Truncated theory with sources}

In this Section we consider the self-accelerated background on which the theory is truncated to the linearized level. As we have discussed before, such a theory is ruled out already by the Solar system data because of the vDVZ discontinuity. Yet, this is the model based on which the conclusions of the instability of the selfaccelerated background have been reached in the literature. As we argued in the previous Sections, the only model that makes physical sense to discuss is the one in which non-linearities are retained, whereas the truncated model has only a marginal interest. Nevertheless, it can be used to discuss certain theoretical questions, such as its relation to the model of massive gravity with the enhanced symmetry [39]. However, even in this case, there are subtle issues concerning the analytic structure and boundary conditions of the Green's functions, which have not be clarified. This issues are important for determining the linearized spectrum of the theory, as we will discuss in detail in the present section.

\section{The square root and boundary conditions}

To get started, let us pick a coordinate system in which the brane is straight and located at the $y=0$ point in the fifth dimension. Let us look at the trace of the 4D 
Einstein equation at the brane without matter or radiation

$$
R=3 m_{c} K
$$

Both sides of (79) are calculated at $y=0^{+}$. The key point is that $K$ can take negative or positive values depending on the boundary conditions in the bulk. This could be seen, e.g., in a gauge in which $K_{\mu \nu} \propto \partial_{y} g_{\mu \nu}(x, y)$, where $g_{\mu \nu}(x, y)$ denotes the $\{\mu \nu\}$ components of the 5D metric. If the metric falls off the brane in the $y$ direction, then the r.h.s. of (79) could be negative. We call this the $(-)$ branch. Alternatively, if the metric grows off the brane in the $y$ direction, the r.h.s. of (179) could be positive. We call this the $(+)$ branch. Clearly, both the $(-)$ and $(+)$ branches admit Minkowski space-time as a solution of (79) . However, the key point is that the $(+)$ branch, in addition, admits the self-accelerated solution.

To get more insight into this set of issues, let us rewrite (79) as a Friedmann equation for a spatially-flat background [2]. It is instructive [51] to discuss the properties of the $(-)$ and $(+)$ branches in terms of this equation. For the $(-)$ branch it takes the form:

$$
H^{2}+m_{c}|H|=0
$$

where $H$ denotes the Hubble parameter as seen by a $4 \mathrm{D}$ brane observer. The solution $H=0$ corresponds to a Minkowski background of the $(-)$ branch. Small perturbations about this space are stable [1].

For the $(+)$ branch, on the other hand, the Friedman equation reads:

$$
H^{2}-m_{c}|H|=0 \text {. }
$$

There is a set of two solutions. The solution $\left(A_{+}\right)$with $H=0$, and $\left(B_{+}\right)$with $|H|=m_{c}$. The $\left(A_{+}\right)$solution is just a Minkowski background of the $(+)$branch. The solution $\left(B_{+}\right)$, that is called the self-accelerated solution [2], is the most interesting one, as it describes an accelerated expansion of the Universe.

In addition to the freedom in choosing boundary conditions in the bulk there is also a freedom in setting a direction of the time arrow $t$ on the brane. This freedom is reflected in the above equations as a symmetry $H \rightarrow-H$. This suggests that a solution with a positive (negative) $H$ could be expanding (contracting) for one choice of the direction of $t$ and contracting (expanding) for the opposite choice.

Some of the above described properties of the $(-)$ and $(+)$ branches can also be seen by analyzing the perturbative amplitudes on a flat space. For them, the operator $\partial_{y}$ in the expression for $K_{\mu \nu}$ is replaced by the square root of the d'Alembertian $\pm \sqrt{-\square_{4}}$. A choice of the bulk boundary conditions is in one-to-one correspondence with the choice of the sign of $\pm \sqrt{-\square_{4}}$. Furthermore, the choice of this sign accompanied with the choice of the direction of the time arrow has to do with the causality and unitarity of the amplitudes; it determines whether there could or could not be tachyon and ghost instabilities in the linearized theory. Since this is an important 
issue, and has direct relevance to our calculations, we will reiterate and sharpen below some of the discussions of Ref. [51.

For the (-) branch, the one-particle exchange amplitude for two very weak sources on the brane (in the momentum space w.r.t. the four worldvolume coordinates) takes the form [1]:

$$
\mathcal{M}^{(-)}(p, y)=\frac{T_{1 / 3}^{2} \exp \left(-\sqrt{p^{2}}|y|\right)}{p^{2}+m_{c} \sqrt{p^{2}}}
$$

where we denote the Euclidean four-momentum squared by $p^{2} \equiv-p_{0}^{2}+p_{1}^{2}+p_{2}^{2}+p_{3}^{2} \equiv$ $p_{4}^{2}+p_{1}^{2}+p_{2}^{2}+p_{3}^{2}$, and

$$
T_{1 / 3}^{2} \equiv 8 \pi G_{N}\left(T_{\mu \nu}^{2}-\frac{1}{3} T_{\alpha}^{\alpha} \cdot T_{\beta}^{\beta}\right) .
$$

The amplitude (82) was constructed by imposing the decreasing boundary conditions in the $y$ direction for positive Euclidean momenta. In the Lorentzian signature this corresponds to an oscillatory dependence on the $y$ coordinate with a plus sign of the exponent $\mathcal{M}^{(-)} \sim \exp \left(+i \sqrt{-p^{2}}|y|\right)$. In the physical domain, $-p^{2} \geq 0$, this corresponds to a retarded amplitude for a given choice of the time direction. Furthermore, there is a branch-cut on the complex plane of $-p^{2}$ on the positive real semi-axes due to the square root in (82), and the pole at $\sqrt{p^{2}}=-m_{c}$ is on a second nonphysical Riemann sheet (see, [51] for detailed discussions.) This pole has a positive residue, while the residue of the pole at $p^{2}=0$ is zero. Thus, it describes a metastable graviton without ghost or tachyon pathologies.

For the $(+)$ branch, on the other hand, the amplitude is determined by choosing the other sign of the square root. As a result, one obtains a Euclidean amplitude that grows with $y$

$$
\mathcal{M}^{(+)}(p, y)=\frac{T_{1 / 3}^{2} \exp \left(\sqrt{p^{2}}|y|\right)}{p^{2}-m_{c} \sqrt{p^{2}}}
$$

In the Lorentzian signature this corresponds to an oscillatory solution with a negative exponent $\mathcal{M}^{(+)} \sim \exp \left(-i \sqrt{-p^{2}}|y|\right)$, and describes an advanced amplitude. The expression (84) differs from (82) also by the position of the pole in the denominator. The pole at $\sqrt{p^{2}}=m_{c}$ is located on a first Riemann sheet on a negative real semi-axes of $-p^{2}$, hence this pole is tachyonic. This suggests that the space-time flat solution of the $(+)$ branch is unstable (this instability could be stronger than tachyonic)! However, the situation can be reversed by replacing the time coordinate $t$, by $-t$ on the $(+)$ branch. Only in this case the Minkowski solution of the $(+)$ branch has a consistent physical interpretation. After this replacement, (84) will describe a retarded amplitude for the new choice of the time direction. Moreover, the tachyonic pole becomes a resonance type pole. This is simply because (84) turns identically into (82). 
On the self-accelerated background of the $(+)$ branch one can calculate two amplitudes with the behavior similar to (82) and (84), as we will show in this Section. However, unlike the flat-space example discussed above, the replacement $t \rightarrow-t$ does not transform one amplitude into the other one.

Summarizing, there are two choices to be made, one for the direction of $y$ and another for $t$. On a Minkowski background these two choices are degenerate. However, on a curved background the degeneracy is lifted and, in general, one should study each particular case separately, as it will be done in this Section.

\section{Spectrum in the presence of a source}

Let us introduce a source $T_{A B}(x, y)=\delta_{A}^{\mu} \delta_{B}^{\nu} T_{\mu \nu}(x) \delta(y)$. In the gauge that we used in Section 2, the source would bend the brane [40. Hence, we define a new coordinate system $(\tilde{x}, \tilde{y})$ in which the brane is straight, and impose the following gauge fixing conditions

$$
\nabla^{\mu} \delta \tilde{g}_{\mu \nu}=\nabla_{\nu} \bar{g}^{\alpha \beta} \delta \tilde{g}_{\alpha \beta}, \quad \delta \tilde{g}_{55}=\delta \tilde{g}_{\mu 5}=0
$$

The new and old coordinate systems, and fields, are related by 5D gauge transformations (41). In terms of the new variables the metric is written as

$$
d s^{2}=\left(\bar{g}_{\mu \nu}+\delta \tilde{g}_{\mu \nu}\right) d \tilde{x}^{\mu} d \tilde{x}^{\nu}+d \tilde{y}^{2} .
$$

Let us look at the equations of motion. The off-diagonal equation (15) written in the new coordinate system is identically satisfied. On the other hand, the $\{55\}$ equation (6) takes the form:

$$
-3 n^{2} \bar{g}^{\mu \nu} \delta \tilde{g}_{\mu \nu}=6 n\left(\bar{g}^{\mu \nu} \delta \tilde{K}_{\mu \nu}-n \bar{g}^{\mu \nu} \delta \tilde{g}_{\mu \nu}\right)
$$

which can be solved to obtain

$$
\bar{g}^{\mu \nu} \delta \tilde{K}_{\mu \nu}=\frac{n}{2} \bar{g}^{\mu \nu} \delta \tilde{g}_{\mu \nu}
$$

The latter is consistent with (41).

The junction condition, taken at $0^{+}$, reads as follows:

$$
-\frac{1}{2}\left(\square_{4} \delta \tilde{g}_{\mu \nu}-\nabla_{\mu} \nabla_{\nu} \delta \tilde{g}_{\alpha}^{\alpha}\right)+2 H^{2} \delta \tilde{g}_{\mu \nu}-\frac{H^{2}}{2} \gamma_{\mu \nu} \delta \tilde{g}_{\alpha}^{\alpha}-H\left(\delta \tilde{K}_{\mu \nu}-\gamma_{\mu \nu} \delta \tilde{K}_{\alpha}^{\alpha}\right)=T_{\mu \nu}
$$

The first four terms on the l.h.s. of (89) is what appears in a $4 \mathrm{D}$ massive gravity at the point of enhanced symmetry (see Section 2 and Appendix B). Here, however, we get additional terms due to the extrinsic curvature (the last two terms on the l.h.s.). This makes the self-accelerated background of the present model different from the massive $4 \mathrm{D}$ gravity at the special point [23]. Because of this, in the present model 
a non-conformal matter stress-tensor is allowed. Indeed, the trace of the junction equation (89) takes the form:

$$
3 m_{c} \bar{g}^{\mu \nu} \delta \tilde{K}_{\mu \nu}=T
$$

(The l.h.s. of this equations would have been zero for a massive $4 \mathrm{D}$ gravity at the special point (23), see Appendix B).

One way to solve for the induced metric $\delta \tilde{g}_{\mu \nu}$ is, to use (41), go to the variables without the tilde sign, and solve first for them. For $\delta g_{\mu \nu}$ we find the following equation (valid at $0^{+}$)

$$
-\frac{1}{2}\left(\square_{4}-4 H^{2}+H \partial_{y}\right) \delta g_{\mu \nu}=T_{\mu \nu}-\frac{1}{3} \gamma_{\mu \nu} T+\Pi_{\mu \nu} \frac{T}{3\left(\square_{4}+4 H^{2}\right)},
$$

where $\Pi_{\mu \nu} \equiv\left(\nabla_{\mu} \nabla_{\nu}+H^{2} \gamma_{\mu \nu}\right)$, and we used $\delta \tilde{K}_{\mu \nu}=\delta K_{\mu \nu}+\Pi_{\mu \nu} \varphi$. Here we note that the last term on the r.h.s. of (91) is somewhat alarming. It already has a pole (all operators are acting from left to right). As a result, the expression for $\delta g_{\mu \nu}$ may end up having a double pole. We will see whether this potential problem can be avoided in the expression for the induced metric $\delta \tilde{g}_{\mu \nu}$.

The trace of the junction condition (90) determines $\varphi$ :

$$
\varphi(x)=\frac{T}{3 m_{c}\left(\square_{4}+4 H^{2}\right)} .
$$

We pause here. The pole in the above expression for $\varphi$ is tachyonic, it corresponds to a scalar of a negative mass square $m_{t}^{2}=-4 H^{2}$. One could conclude that the brane bending mode $\varphi$ is at least as bad as a tachyon. However, $\varphi$ is gauge dependent, and, in order to reach a conclusion one needs to calculate a gauge invariant physical amplitude.

Finally, for future reference we write the expression that encompasses both the bulk and junction equations for $\delta g_{\mu \nu}$ :

$$
\left(H \partial_{y}^{2}+\left\{\frac{H}{A^{2}}+2 \delta(y)\right\}\left(\square_{4}-4 H^{2}\right)\right) \delta g_{\mu \nu}=-4\left(T_{\mu \nu}-\frac{1}{3} \gamma_{\mu \nu} T+H \Pi_{\mu \nu} \varphi\right) \delta(y) .
$$

We will use the above equation for the determination of the induced metric in the next subsection. The reminder of this subsection discusses a certain subtlety that emerges in the above equation. A reader who is not interested in these details could skip to the next subsection, where the actual calculations are performed.

This concerns the last term in the parenthesis on the r.h.s. of (93) (we will call it $\left.\Delta_{\mu \nu}\right)$ :

$$
\Delta_{\mu \nu} \equiv\left(\nabla_{\mu} \nabla_{\nu}+H^{2} \gamma_{\mu \nu}\right) \frac{1}{\square_{4}+4 H^{2}} \frac{T}{3}
$$

Looking at (94) one may conclude that it has a tachyonic pole at $\square_{4}+4 H^{2}=0$. However, this would be a misleading conclusion. To see this we use the identity

$$
\left.\left(\square_{4}-4 H^{2}\right)\left(\nabla_{\mu} \nabla_{\nu}+H^{2} \gamma_{\mu \nu}\right)(\text { scalar })=\left(\nabla_{\mu} \nabla_{\nu}-H^{2} \gamma_{\mu \nu}\right)\left(\square_{4}+4 H^{2}\right) \text { (scalar }\right),
$$


which allows us to rewrite $\Delta_{\mu \nu}$ as:

$$
\Delta_{\mu \nu} \equiv \frac{1}{\square_{4}-4 H^{2}}\left(\nabla_{\mu} \nabla_{\nu}-H^{2} \gamma_{\mu \nu}\right) \frac{T}{3} .
$$

As it is clear from (96), the expression for $\Delta_{\mu \nu}$ seems now to have a pole at $\square_{4}-$ $4 H^{2}=0$, for the operators that multiply it from the left. We will carefully deal with such subtleties in the next subsection.

\section{Poles and residues}

In order to find the physical spectrum of the theory one should look at the poles and residues of the induced metric:

$$
\delta \tilde{g}_{\mu \nu}(x, y)=\delta g_{\mu \nu}(x, y)+\frac{2 A}{H} \Pi_{\mu \nu} \varphi
$$

Substituting (97) into (91), and using the identity (95) we find the equation

$$
-\frac{1}{2}\left(\square_{4}-4 H^{2}+H \partial_{y}\right) \delta \tilde{g}_{\mu \nu}=T_{\mu \nu}-\frac{1}{3} \gamma_{\mu \nu} T-\left(\nabla_{\mu} \nabla_{\nu}-H^{2} \gamma_{\mu \nu}\right) \frac{T}{3 H^{2}} .
$$

As before, this equation is written for $\tilde{y}=0^{+}$. Note, that the troublesome doublepole structure that was present in (91), has remarkably canceled out in the above equation.

In order to find the expression for $\delta \tilde{g}_{\mu \nu}$ that would capture both the bulk and junction equations, we use (97) in (93). The resulting equations for $\delta \tilde{g}_{\mu \nu}$ reads

$$
\begin{array}{r}
\left(H \partial_{y}^{2}+\left\{\frac{H}{A^{2}}+2 \delta(y)\right\}\left(\square_{4}-4 H^{2}\right)\right) \delta \tilde{g}_{\mu \nu}= \\
-4\left(T_{\mu \nu}-\frac{1}{3} \gamma_{\mu \nu} T-\left(\nabla_{\mu} \nabla_{\nu}-H^{2} \gamma_{\mu \nu}\right) \frac{T}{3 H^{2}}\right) \delta(y)+\frac{2}{A}\left(\nabla_{\mu} \nabla_{\nu}-H^{2} \gamma_{\mu \nu}\right) \frac{T}{3 H} .
\end{array}
$$

Here we would like to emphasize that the last term on the r.h.s. of (99) does not vanish outside of the brane. Therefore, even a source that is localized on the brane, gives rise to an effective source that is delocalized all over the bulk. It is the effective source that excites the physical metric $\delta \tilde{g}_{\mu \nu}$. Moreover, this effective source grows in the bulk (this is because in physical amplitudes the last term on the r.h.s. of (99) should be multiplied by an additional factor of $A^{2}$ arising from the norm of the inner product). This rather unusual behavior is a consequence of the fact that in the coordinate system in which the brane is static and straight the background itself grows in the bulk as $A^{2}$.

Let us now solve (99). It yields two independent solutions

$$
\begin{aligned}
\frac{1}{2} \delta \tilde{g}_{\mu \nu \pm}= & \frac{1}{\mathcal{O}^{(4)}} A^{\alpha_{ \pm}\left(\mathcal{O}^{(4)}\right)} \frac{1}{\mathcal{O}^{(4)}-H^{2} \alpha_{ \pm}\left(\mathcal{O}^{(4)}\right)}\left\{\mathcal{O}^{(4)}\left(T_{\mu \nu}-\frac{1}{3} \gamma_{\mu \nu} T\right)-\Pi_{\mu \nu}^{-} \frac{T}{3}\right\} \\
& -\frac{1}{3 H^{2}} \frac{A}{\mathcal{O}^{(4)}} \Pi_{\mu \nu}^{-} T
\end{aligned}
$$


where $\mathcal{O}^{(4)}, \Pi_{\mu \nu}^{-}$and $\alpha_{ \pm}$are respectively defined by

$$
\begin{aligned}
\mathcal{O}^{(4)} & =-\square_{4}+4 H^{2}, \\
\Pi_{\mu \nu}^{-} & =\nabla_{\mu} \nabla_{\nu}-H^{2} \gamma_{\mu \nu}, \\
\alpha_{ \pm}(z) & =\frac{1}{2}\left(1 \pm \sqrt{1+\frac{4 z}{H^{2}}}\right) .
\end{aligned}
$$

It is important to point out that the r.h.s. of (100) has potentially two poles: the first one for the zero eigenvalues of $\mathcal{O}^{(4)}$ and the second for zero eigenvalues of $\mathcal{O}^{(4)}-H^{2} \alpha_{ \pm}\left(\mathcal{O}^{(4)}\right)$. These poles and their residues will be discussed in detail below.

Equations (100) can be rewritten as

$$
\begin{aligned}
\frac{1}{2} \delta \tilde{g}_{\mu \nu \pm}= & K_{ \pm}\left(\mathcal{O}^{(4)}\right)\left[T_{\mu \nu}^{T T}+\frac{1}{3}\left(P_{\mu \nu} \frac{1}{\mathcal{Q}^{(4)}}-\frac{1}{\mathcal{O}^{(4)}} P_{\mu \nu}\right) T\right] \\
& -\frac{A}{3 H^{2} \mathcal{O}^{(4)}} P_{\mu \nu} T+\frac{A}{12 H^{2}} \gamma_{\mu \nu} T,
\end{aligned}
$$

where $P_{\mu \nu}=\nabla_{\mu} \nabla_{\nu}-\frac{1}{4} \gamma_{\mu \nu} \square_{4}$ (i.e., $\left.\Pi_{\mu \nu}^{-}=P_{\mu \nu}-\frac{1}{4} \gamma_{\mu \nu} \mathcal{O}^{(4)}\right), \mathcal{Q}^{(4)}=-\square_{4}-4 H^{2}=$ $\mathcal{O}^{(4)}-8 H^{2}$

$$
K_{ \pm}(z)=\frac{A^{\alpha_{ \pm}}(z)}{z-H^{2} \alpha_{ \pm}(z)},
$$

and the decomposition of a covariantly conserved symmetric tensor in terms of its transverse-traceless, spin-0 longitudinal, and pure trace parts was used, namely,

$$
T_{\mu \nu}=T_{\mu \nu}^{T T}+\frac{1}{4} \gamma_{\mu \nu} T+\frac{1}{3} P_{\mu \nu} \frac{1}{\mathcal{Q}^{(4)}} T .
$$

Noticing that

$$
P_{\mu \nu}\left(\square_{4}+8 H^{2}\right) \varphi=\square_{4} P_{\mu \nu} \varphi,
$$

for any scalar $\varphi$ (which implies that $P_{\mu \nu} f\left(\mathcal{Q}^{(4)}\right) \varphi=f\left(\mathcal{O}^{(4)}\right) P_{\mu \nu} \varphi$ for any smooth function $f$ ) the term in the parenthesis in (104) vanishes.

Next, let us introduce the Lichnerowicz operator $\Delta_{L}$ with the following properties

$$
\begin{aligned}
\left(\Delta_{L}-4 H^{2}\right) T_{\mu \nu}^{T T} & =\mathcal{O}^{(4)} T_{\mu \nu}^{T T}, \\
\left(\Delta_{L}-4 H^{2}\right) \gamma_{\mu \nu} \varphi & =\gamma_{\mu \nu} \mathcal{Q}^{(4)} \varphi, \\
\left(\Delta_{L}-4 H^{2}\right) P_{\mu \nu} \varphi & =P_{\mu \nu} \mathcal{Q}^{(4)} \varphi,
\end{aligned}
$$

for an arbitrary scalar $\varphi$. From (104) we obtain

$$
\begin{aligned}
\frac{1}{2} \delta \tilde{g}_{\mu \nu \pm}= & K_{ \pm}\left(\Delta_{L}-4 H^{2}\right) T_{\mu \nu}^{T T}-\frac{A}{3 H^{2}} P_{\mu \nu} \frac{1}{\mathcal{Q}^{(4)}} T+\frac{A}{12 H^{2}} \gamma_{\mu \nu} T \\
= & K_{ \pm}\left(\Delta_{L}-4 H^{2}\right) T_{\mu \nu}+\frac{1}{12} \gamma_{\mu \nu}\left(\frac{\square_{4}}{\mathcal{Q}^{(4)}}-3\right) K_{ \pm}\left(\mathcal{Q}^{(4)}\right) T-\frac{A}{3} \gamma_{\mu \nu} \frac{1}{\mathcal{Q}^{(4)}} T \\
& -\frac{1}{3} \nabla_{\mu} \nabla_{\nu}\left(K_{ \pm}\left(\mathcal{Q}^{(4)}\right)+\frac{A}{H^{2}}\right) \frac{1}{\mathcal{Q}^{(4)}} T
\end{aligned}
$$


To get to the second line of (109) we expressed $T_{\mu \nu}^{T T}$ in terms of $T_{\mu \nu}$ and $T$ using (106) and the properties (108) of $\Delta_{L}$. In the last term of the third line, the tachyonic pole $\mathcal{Q}^{(4)}=0$ could lead to a boundary divergence, impeding integration by parts when we contract with a conserved $T_{\mu \nu}^{\prime}$.

Below we discuss the two cases of the $(-)$ and $(+)$ solutions separately. In the (-) case (this is the only case that has been so far discussed in the literature), there is the double pole present in the Green's function. Usually a double pole can be recast into a sum/difference of two simple poles, giving rise to ghosts or tachyons. In the present case, this decomposition cannot be done so clearly. This is because nonlocal operators (such a square roots of the covariant d'Alembertian) are involved. The residue of the double pole diverges, which may or may not be a signal of a singularity of the perturbative approach at scales $\sim H$. On the other hand, the repulsive nature of the additional contributions (as compared to massive gravity) may be suggestive of a ghost-like state. However, given the results of Section 2, it is not entirely clear whether this ghost-like state, even if present, can be emitted in a final state, or it only appears as an intermediate state. In the latter case it would have been harmless. To clarify this, one needs a BRST invariant construction of the physical Hilbert space. Since the truncated theory considered here has only a limited interest anyway, we won't pursue this tedious project. One is certain, the conclusion reached in the literature that the amplitude has a ghost, is not entirely obvious beyond a reasonable doubt.

For the $(+)$ solution, the $1 / \mathcal{Q}^{(4)}$ divergence is not present since $K_{+}\left(\mathcal{Q}^{(4)}\right)+A / H^{2}$ also vanishes in the $\mathcal{Q}^{(4)} \rightarrow 0$ limit. Therefore, integrating on both sides of (109) $\int d^{4} x \sqrt{\gamma} T_{\mu \nu}^{\prime} \cdots$, for the $(+)$ solution, (and integrating by parts the last term)

$$
\begin{aligned}
\frac{1}{2} \int d^{4} x \sqrt{\gamma} T^{\prime \mu \nu} \delta \tilde{g}_{\mu \nu_{+}}= & \int d^{4} x \sqrt{\gamma}\left(T^{\prime \mu \nu} K_{+}\left(\Delta_{L}-4 H^{2}\right) T_{\mu \nu}\right. \\
& \left.+\frac{1}{12} T^{\prime}\left(\frac{\square_{4}}{\mathcal{Q}^{(4)}}-3\right) K_{+}\left(\mathcal{Q}^{(4)}\right) T-\frac{A}{3} T^{\prime} \frac{1}{\mathcal{Q}^{(4)}} T\right)
\end{aligned}
$$

The pole at $\mathcal{Q}^{(4)}=0$ has zero residue, and the residue of the pole at $\mathcal{Q}^{(4)}=$ $2 H^{2}\left(\square_{4}=-6 H^{2}\right)$ together with the one at $\Delta_{L}=6 H^{2}$ give a massless graviton amplitude! Both, the tensorial structure in the numerator and, very importantly, the pole in the denominator, correspond to a propagating massless spin-2 state on dS background. What is this massless state? It corresponds to non-normalizable zero-mode discussed in Section 2. To understand this result better, it is instructive to look at the spectral representation of the amplitude (110). Let us rewrite (110) as

$$
\mathcal{A}=\int T^{\prime \mu \nu} K_{+}\left(\Delta_{L}-4 H^{2}\right) T_{\mu \nu}-\frac{1}{3} T^{\prime} K_{+}(\mathcal{Q}) T-\frac{1}{3} T^{\prime} \frac{H^{2}}{\mathcal{Q}} K_{+}(\mathcal{Q}) T-\frac{1}{3} T^{\prime} \frac{1}{\mathcal{Q}} T .(1
$$

Which can be compared with the amplitude for massive gravity on de Sitter space 
found in [52]:

$$
\begin{aligned}
\mathcal{A}_{M}= & \int T^{\prime \mu \nu}\left(\Delta_{L}-4 H^{2}-M^{2}-2 H^{2}\right)^{-1} T_{\mu \nu}-\frac{1}{3} T^{\prime}\left(\mathcal{Q}+M^{2}-2 H^{2}\right)^{-1} T- \\
& \frac{1}{3} T^{\prime} \frac{H^{2}}{\mathcal{Q}}\left(\mathcal{Q}+M^{2}-2 H^{2}\right)^{-1} T-\frac{H^{2}}{6 H^{2}-3 M^{2}} T^{\prime} \frac{1}{\mathcal{Q}} T .
\end{aligned}
$$

It is sufficient at this point to obtain the spectral representation at $y=0$ which is completely determined by the function $K_{+}(z)$ for the first two terms in the r.h.s of (111). Below, $z$ will refer to $\mathcal{Q}$, and we will define the complex coordinate $w=$ $-H^{2} / 4-z$ which in the flat space-time limit is positive in the physical domain, i.e., $w \rightarrow-p^{2} \geq 0$. Therefore, we study the spectral decomposition of $\tilde{K}_{+}(w)=$ $K_{+}\left(-w-\frac{H^{2}}{4}\right)$ in the complex $w$ plane.

$$
\tilde{K}_{+}(w)=\frac{1}{-w-\frac{H^{2}}{4}-\frac{H m_{c}}{2}\left(1+\frac{2}{H} \sqrt{-w}\right)},
$$

where we have restored $m_{c}$ to be able to take the flat limit keeping $m_{c}$ finite. The flat limit of this expression, $\tilde{K}_{+}^{0}(w)$, gives the flat propagator of the $(+)$ branch described before:

$$
\tilde{K}_{+}^{0}(w)=\frac{1}{-w-m_{c} \sqrt{-w}}
$$

This flat propagator can be decomposed as a sum over KK contributions with spectral density $\rho$ via a contour integral which goes around the cut just below and above the $w$ positive real semi-axis and closes at infinity through a counterclockwise circle (the only contribution coming from the jump of the imaginary part of $K$ across the cut, $\Delta \operatorname{Im} K)$ :

$$
\tilde{K}_{+}^{0}(w)=\int_{0}^{\infty} \frac{d s}{s-w} \rho(s)=\frac{1}{2 \pi} \int_{0}^{\infty} \frac{d s}{s-w} \Delta \operatorname{Im} K(s)=\frac{1}{\pi} \int_{0}^{\infty} \frac{d s}{s-w} \frac{m_{c} \sqrt{s}}{s^{2}+m_{c}^{2} s},
$$

where (114) has to be interpreted as

$$
\tilde{K}_{+}^{0}(w)=\frac{1}{-w-m_{c} \sqrt{\mathrm{e}^{i \pi} w}}=\frac{1}{-w-i m_{c} \sqrt{w}},
$$

that implies that there is no pole for $w=|w| \mathrm{e}^{i \phi}$ in the first Riemann sheet, i.e., $0 \leq \phi<2 \pi$. The state corresponding to the pole of the propagator (114) at $w=\mathrm{e}^{-i \pi} m_{c}^{2}$ is on the second Riemann sheet and does not contribute to physical amplitudes. Therefore, by a suitable prescription (which amounts to a choice of time direction) we are able to find an amplitude with no tachyonic instabilities.

However, in trying to extend this argument to the curved background case, we face a problem. The curved generalization for (113) yields the following spectral representation:

$$
\tilde{K}_{+}(w)=-\frac{2}{4 w+H^{2}}+\frac{1}{\pi} \int_{0}^{\infty} \frac{d s}{s-w} \frac{16 m_{c} \sqrt{s}}{\left(H\left(H+2 m_{c}\right)+4 s\right)^{2}+4 m_{c}^{2} s} .
$$


As in the flat case, the zero mode from the pole at $w=\mathrm{e}^{-i \pi}\left(H+2 m_{c}\right)^{2} / 4$ (corresponding to $z=2 H^{2}$ when $m_{c}=H$ ) does not contribute in (117) while the KK's $0 \leq w_{K K}<\infty\left(-H^{2} / 2 \leq z_{K K}<\infty\right)$ and the isolated mode $w=\mathrm{e}^{i \pi} H^{2} / 4$ do. This prescription, also made the flat amplitude tachyon free, has turned the curved amplitude into the one of the $(-)$ branch with a ghostlike double pole in the third term of (111).

In order to describe the $(+)$ branch amplitude, with only a massless pole an a branch cut of KK's, we must consider the other possible prescription, i.e., $\sqrt{-w} \equiv$ $\sqrt{\mathrm{e}^{-i \pi} w}$, the decomposition is then

$$
\tilde{K}_{+}(w)=-\frac{6}{4 w+\left(H+2 m_{c}\right)^{2}}-\frac{1}{\pi} \int_{0}^{\infty} \frac{d s}{s-w} \frac{16 m_{c} \sqrt{s}}{\left(H\left(H+2 m_{c}\right)+4 s\right)^{2}+4 m_{c}^{2} s} .
$$

Now, the zero mode from the pole of (113) at $w=\mathrm{e}^{i \pi}\left(H+2 m_{c}\right)^{2} / 4$ is on the first Riemann sheet and does contribute to the decomposition and the continuum enters with opposite sign. This relative sign implies that once the KK modes are treated as conventional positive-residue states, the residue of the zero mode has to be negative. This would imply that the zero-mode is a ghost. However, the zero-mode is not a normalizable state and because of this its interpretation is obscure. In particular, whether or not it can be emitted in a final state by any mater source localized on the brane is not clear.

\section{Outlook}

We studied the question of small perturbations on the self-accelerated solution of the DGP model. The issue is rather involved. We showed that the small perturbations on an empty self-accelerated background can be quantized without yielding ghosts or other instabilities. For conformal sources, such as radiation, small perturbations are instability free as well. More realistic, non-conformal sources, however, could trigger ghost-like instabilities in the linearized theory, and become unstable. We have suggested, in Section 4, possible loopholes in the latter conclusion, even within the linearized theory itself. More importantly, however, those non-conformal sources lead also to the breakdown of the linearized calculations, and, therefore, no conclusion on the (in)stability of the solution can be drawn without invoking non-linear dynamics. For the available non-linear spherically symmetric solution the dangerous linearized modes are spurious.

To establish stability of generic non-conformal sources, one has to perform calculations of 5D perturbations about a background produced by the source itself, since the latter is dominating over the cosmological background locally. One should see which of the obtained perturbations could be matched, at the scale $r_{*}$, to solutions in the far-away region. Further interesting question is to study deviations from the self-accelerated background by introducing a small cosmological constant to explore the spectrum of the theory. We hope to report on some of these issues elsewhere. 


\section{Acknowledgments}

We would like to thank G. Dvali, G. Esposito-Farése, T. Gherghetta, M. Kleban, N. Kaloper, K. Koyama, P. Mannheim, J. Mourad, M. Porrati, M. Redi, R. Scoccimarro, T. Tanaka, and M. Zaldarriaga for useful discussions. Work of GG and AI is supported in part by NASA grant NNGG05GH34G and in part by NSF grant 0403005. CD would like to thank CCPP at New York University, and GG to thank the Weizmann Institute, for hospitality, where parts of this work were done.

\section{Appendix A}

The goal of the present section is to discuss a toy model of a real scalar field minimally coupled to a gravitational self-accelerated background. The idea is that the spectrum of small perturbations of this scalar bears some similarities to that of the gravitational perturbations. The action of the toy model is

$$
S=\int d^{4} x d y \sqrt{g}\left(-\frac{1}{2} g^{A B} \partial_{A} \Phi \partial_{B} \Phi\right)+2 r_{c} \int d^{4} x \sqrt{\gamma}\left(-\frac{1}{2} \gamma^{\mu \nu} \partial_{\mu} \varphi \partial_{\nu} \varphi\right)
$$

where $A, B=0,1,2,3,5, \mu, \nu=0,1,2,3, \varphi(x) \equiv \Phi(x, y=0), \gamma_{\mu \nu}(x)=g_{\mu \nu}(x, y=$ $0)$, and the metric of the spatially-flat self-accelerated background is [2]

$$
d s^{2}=(1+H|y|)^{2}\left(-d t^{2}+a^{2}(t) d \vec{x}^{2}\right)+d y^{2} \equiv A^{2}(y) \gamma_{\mu \nu}(x) d x^{\mu} d x^{\nu}+d y^{2} .
$$

The equation of motion reads:

$$
\partial_{A}\left(\sqrt{g} g^{A B} \partial_{B} \Phi\right)+2 r_{c} \delta(y) \partial_{\mu}\left(\sqrt{\gamma} \gamma^{\mu \nu} \partial_{\nu} \varphi\right)=0
$$

Since $\sqrt{g}=A^{4} a^{3}$ and $\sqrt{\gamma}=a^{3}$ we obtain for the bulk equation:

$$
\frac{1}{\sqrt{\gamma}} \partial_{\mu}\left(\sqrt{\gamma} \gamma^{\mu \nu} \partial_{\nu} \Phi\right) \equiv \square_{4} \Phi=-\frac{\partial_{y}\left(A^{4} \partial_{y} \Phi\right)}{A^{2}}
$$

Moreover, for the junctions condition we get

$$
\left[\partial_{y} \Phi\right]_{-\epsilon}^{+\epsilon}=-\left.2 r_{c} \square_{4} \Phi\right|_{0} .
$$

We look for the KK modes that are solutions to (122) and (123) and can be expressed in the form:

$$
\Phi(x, y) \equiv \int \varphi_{m}(x) f_{m}(y) d m, \quad \square_{4} \varphi_{m}(x)=m^{2} \varphi_{m}(x) .
$$

For the KK modes the bulk equation (122) and the junction condition (123) can be rewritten as follows:

$$
m^{2} f_{m}(y)=-\frac{\partial_{y}\left(A^{4} \partial_{y} f_{m}\right)}{A^{2}}
$$


and

$$
\left[\partial_{y} f_{m}(y)\right]_{-\epsilon}^{+\epsilon}=-2 r_{c} m^{2} f_{m}(0)
$$

As in the graviton case, and because of the boundary condition that depends on the mass of a KK mode (126), the modes themselves are not orthogonal.

To solve the above equations it is useful to make the following change of variables from $y$ to $z$, and introduce a new functions $u_{m}$ :

$$
d z \equiv \frac{d y}{A(y)}, \quad f_{m} \equiv A^{-3 / 2} u_{m}
$$

In terms of the new coordinate $A(z)=\exp (H|z|)$, and $\partial_{y}=A^{-1}(z) \partial_{z}$. As a result, the bulk equation takes the form:

$$
-\frac{d^{2} u_{m}}{d z^{2}}+\left(\frac{9 H^{2}}{4}-m^{2}\right) u_{m}=0
$$

The junctions condition (126) rewritten in terms of $u_{m}$ reads:

$$
\left[\partial_{z} u_{m}(z)\right]_{-\epsilon}^{+\epsilon}=\left(3 H-2 r_{c} m^{2}\right) u_{m}(0)
$$

From the above two equations it is immediately clear that there is a zero-mode solution $m=0, u=\exp (3 H|z| / 2)$. However, this mode is diverging in the bulk.

Other solutions of the above system of equations should be supplemented by the normalization condition, which in terms of the old and new variables reads as follows:

$$
\int_{-\infty}^{+\infty} d y \frac{\sqrt{g}}{\sqrt{\gamma}} g^{00} f_{m}^{2}(y)=\int_{-\infty}^{+\infty} d z u_{m}^{2}(z)<\infty .
$$

Let us analyze the above system of equations. It is straightforward to check that there exists only one normalizable solution:

$$
u_{m_{*}}=\sqrt{\alpha} e^{-\alpha|z|}, \quad \alpha \equiv \frac{3 H}{2}-m_{c},
$$

while the mass of this mode is

$$
m_{*}^{2}=3 H m_{c}-m_{c}^{2} .
$$

The above corresponds to the self-accelerated background if $H=m_{c}$. Then, we get $\alpha=H / 2, m_{*}^{2}=2 H^{2}$, and $u_{m_{*}}=(H / 2 A)^{1 / 2}$.

What are the other solutions of (128) and (129)? The rest of the solutions constitute a continuum of massive states with $m^{2} \geq 9 H^{2} / 4$ with the plane-wave normalizable wavefunctions:

$$
u_{m}(z)=A \sin (\omega|z|)+B \cos (\omega z), \quad \omega \equiv \sqrt{m^{2}-\frac{9 H^{2}}{4}},
$$


where the normalization coefficients $A$ and $B$ can be calculated

$$
A=-B \frac{2 m^{2}-3 m_{c} H}{m_{c} \omega},
$$

while the expression for $B$ determines an important dependence of the wavefunctions on $m^{2}$ on the brane, in analogy with the flat case [53].

\section{Appendix B}

In this appendix we consider linearized $4 \mathrm{D}$ massive gravity about a dS space with the special relation between the mass and dS curvature given in (23). The Lagrangian density takes the form (see, e.g., [42], but the overall coefficient is different here)

$$
\begin{array}{r}
-\frac{1}{2} \mathcal{L}_{*}=\frac{1}{2}\left(\nabla_{\mu} h^{\mu \alpha}\right)^{2}+\frac{1}{4} h_{\mu \nu} \square_{4} h^{\mu \nu}-\frac{1}{4} h \square_{4} h+\frac{1}{2} h^{\mu \nu} \nabla_{\mu} \nabla_{\nu} h \\
-\frac{1}{2} H^{2}\left(h_{\mu \nu}^{2}+\frac{1}{2} h^{2}\right)-\frac{1}{4} m_{*}^{2}\left(h_{\mu \nu}^{2}-h^{2}\right) .
\end{array}
$$

For a generic value of the graviton mass the above Lagrangian has no gauge symmetry. However, for a special value (23) there is a symmetry present:

$$
\delta h_{\mu \nu}(x)=\left(\nabla_{\mu} \nabla_{\nu}+H^{2} \gamma_{\mu \nu}\right) \rho(x) .
$$

The above gauge transformation allows one to gauge-remove one out of five on-shell polarizations of the massive graviton [39. However, this theory does not admit a coupling to non-conformal matter. There are many ways to see this. The most straightforward one is to write down the equations of motion, use the Bianchi identities, and observe that the trace equation would necessarily imply $T_{\mu}^{\mu}=0$. This can also be established by noticing that introduction of the coupling $h_{\mu \nu} T^{\mu \nu}$ in the Lagrangian (135) violates the symmetry (136) unless $T_{\mu}^{\mu}=0$. Then, one could introduce a coupling a la Stückelberg $\left(h_{\mu \nu}-\left(\nabla_{\mu} \nabla_{\nu}+H^{2} \gamma_{\mu \nu}\right) \sigma\right) T^{\mu \nu}$, where $\sigma$ is a field that also transforms as $\delta \sigma(x)=\rho(x)$ when the metric is transformed according to (136). Such a coupling would not violate the symmetry of the theory. However, the equation of motion of the $\sigma$ field would require that $T_{\mu}^{\mu}=0$. As we have seen in the Section 2, in the DGP model this difficulty is avoided due to the extrinsic curvature terms.

To this end we would like to calculate the propagator of the theory given by (135) without coupling it to any matter. The gauge independent part of the propagator would tell us how many degrees of freedom are propagating in the theory. Without introducing the source, the propagator is defined as an inverse of the quadratic operator in (135). However, because of the symmetry (136), this operator is not invertible. Hence, we should proceed by introducing the gauge fixing term which we choose to be

$$
\mathcal{L}_{\text {gf }}=-\frac{1}{\alpha} h^{2} .
$$


After this term is included, we introduce a "spectator" $T_{\mu \nu}$, for which $T \neq 0$. Formally, this is possible because of the gauge fixing term. However we should keep in mind that the original theory wouldn't admit any non-conformal matter, and, in that respect, the introduction of the "spectator" non-conformal source after the gauge fixing is just a technical trick that enables one to extract the non-derivative terms of the propagator without too much of calculations. We follow this philosophy below.

In this gauge the equations of motion read:

$$
\begin{aligned}
\frac{1}{2}\left(\square_{4} h_{\mu \nu}-\right. & \left.\nabla_{\nu} \nabla_{\alpha} h_{\mu}^{\alpha}-\nabla_{\mu} \nabla_{\alpha} h_{\nu}^{\alpha}+\nabla_{\mu} \nabla_{\nu} h\right)+\frac{1}{2} \gamma_{\mu \nu}\left(\nabla_{\alpha} \nabla_{\beta} h^{\alpha \beta}-\square_{4} h\right) \\
- & H^{2}\left(h_{\mu \nu}+\frac{1}{2} \gamma_{\mu \nu} h\right)-\frac{m_{*}^{2}}{2}\left(h_{\mu \nu}-\gamma_{\mu \nu} h\right)+\frac{1}{\alpha} \gamma_{\mu \nu} h=-T_{\mu \nu} .
\end{aligned}
$$

The Bianchi identities enforce the following constraint:

$$
\frac{m_{*}^{2}}{2}\left(\nabla^{\mu} h_{\mu \nu}-\nabla_{\nu} h\right)=-\frac{1}{\alpha} \nabla_{\nu} h .
$$

The trace of equation (138) takes the form

$$
\nabla^{\mu} \nabla^{\nu} h_{\mu \nu}-\square_{4} h+3\left(\frac{m_{*}^{2}}{2}-H^{2}\right) h+\frac{4}{\alpha} h=-T .
$$

The above system of equations can be solved. In what follows we concentrate on the case when (23) is satisfied, i.e., $m_{*}^{2}=2 H^{2}$. First we solve for the trace

$$
h=\frac{\alpha T H^{2}}{\square_{4}-4 H^{2}} .
$$

As we see, in a theory without the gauge fixing term (i.e, when $\alpha \rightarrow \infty$ ) the field diverges.

Next using the identity (95) we solve for $h_{\mu \nu}$ :

$$
-\frac{1}{2} h_{\mu \nu}=\frac{T_{\mu \nu}-\frac{1}{2} \gamma_{\mu \nu} T}{\square_{4}-4 H^{2}}+\left(1-\frac{\alpha H^{2}}{2}\right) \frac{1}{\left(\square_{4}-4 H^{2}\right)}\left(\nabla_{\mu} \nabla_{\nu}-H^{2} \gamma_{\mu \nu}\right) \frac{T}{\left(\square_{4}-4 H^{2}\right)}(1 .
$$

Fortunately, the double pole terms on the r.h.s. are gauge dependent ${ }^{19}$. On the other hand, the gauge-independent part of the above expression gives the non-derivative part of the inverse of the gauge kinetic term of (135). The latter takes the form

$$
\frac{1}{2} \frac{\gamma_{\mu \alpha} \gamma_{\nu \beta}+\gamma_{\mu \beta} \gamma_{\nu \alpha}-\gamma_{\mu \nu} \gamma_{\alpha \beta}}{\square_{4}-4 H^{2}}+\ldots
$$

The tensorial structure in the numerator of Eq. (143) confirms that the theory (135) at the linearized level does not propagate the fifth, helicity-0, degree of freedom [39.

\footnotetext{
${ }^{19}$ Notice that the gauge-dependent terms in this case contain not only derivatives but also terms proportional to $\gamma_{\mu \nu}$. This is a direct consequence of the form of the gauge transformations (136).
} 


\section{Appendix C}

In this appendix we give the expressions used in Section 3 for the first and second order perturbations of the Ricci tensor around a de Sitter background metric $\gamma_{\mu \nu}$.

The metric is expanded as

$$
g_{\mu \nu}=\gamma_{\mu \nu}+\delta g_{\mu \nu}
$$

with inverse

$$
g^{\mu \nu}=\gamma^{\mu \nu}-\delta g^{\mu \nu}+\delta g_{\rho}^{\mu} \delta g^{\rho \nu}
$$

The first order Ricci tensor components are given by

$$
R_{\mu \nu}^{(1)}(\delta g)=-\frac{1}{2}\left(\square \delta g_{\mu \nu}-\nabla_{\alpha} \nabla_{\mu} \delta g_{\nu}^{\alpha}-\nabla_{\alpha} \nabla_{\nu} \delta g_{\mu}^{\alpha}+\nabla_{\mu} \nabla_{\nu} \delta g_{\alpha}^{\alpha}\right)
$$

which for a transverse traceless perturbation, $\delta g_{\mu \nu}=h_{\mu \nu}^{T T}$, reduces to

$$
R_{\mu \nu}^{(1)}\left(h^{T T}\right)=-\frac{1}{2} \square h_{\mu \nu}^{T T}+4 H^{2} h_{\mu \nu}^{T T} .
$$

Note that the $\nabla_{\alpha}$ has to be commuted through $\nabla_{\mu, \nu}$ in the right hand side of (146) to get (147). Here, we recall that for the de Sitter background metric $\gamma_{\mu \nu}$ we have $\left[\nabla_{\alpha}, \nabla_{\mu}\right] h_{\nu}^{\alpha}=4 H^{2} h_{\mu \nu}-H^{2} \gamma_{\mu \nu} h_{\alpha}^{\alpha}$.

The second order terms are given by

$$
\begin{aligned}
R_{\mu \nu}^{(2)}(\delta g)= & +\frac{1}{4}\left(\nabla^{\rho} \delta g_{\alpha}^{\alpha}-2 \nabla^{\alpha} \delta g_{\alpha}^{\rho}\right)\left(\nabla_{\mu} \delta g_{\nu \rho}+\nabla_{\nu} \delta g_{\mu \rho}-\nabla_{\rho} \delta g_{\mu \nu}\right) \\
& -\frac{1}{4}\left(\nabla^{\rho} \delta g_{\mu}^{\alpha}-\nabla_{\mu} \delta g^{\rho \alpha}-\nabla_{\alpha} \delta g_{\mu}^{\rho}\right)\left(\nabla_{\alpha} \delta g_{\nu \rho}+\nabla_{\nu} \delta g_{\alpha \rho}-\nabla_{\rho} \delta g_{\nu \alpha}\right) \\
& -\frac{1}{2} \delta g^{\alpha \rho}\left(\nabla_{\alpha} \nabla_{\mu} \delta g_{\rho \nu}+\nabla_{\alpha} \nabla_{\nu} \delta g_{\rho \mu}-\nabla_{\alpha} \nabla_{\rho} \delta g_{\mu \nu}\right) \\
& +\frac{1}{2} \delta g^{\alpha \rho}\left(\nabla_{\mu} \nabla_{\alpha} \delta g_{\rho \nu}+\nabla_{\mu} \nabla_{\nu} \delta g_{\rho \alpha}-\nabla_{\mu} \nabla_{\rho} \delta g_{\nu \alpha}\right)
\end{aligned}
$$

Which for a transverse traceless perturbation reads (we drop the TT superscript on the r.h.s.)

$$
\begin{array}{r}
R_{\mu \nu}^{(2)}\left(h^{T T}\right)=-\frac{1}{4}\left(\nabla^{\rho} h_{\mu}^{\alpha}-\nabla_{\mu} h^{\rho \alpha}-\nabla_{\alpha} h_{\mu}^{\rho}\right)\left(\nabla_{\alpha} h_{\nu \rho}+\nabla_{\nu} h_{\alpha \rho}-\nabla_{\rho} h_{\nu \alpha}\right) \\
-\frac{1}{2} h^{\alpha \rho}\left(\nabla_{\alpha} \nabla_{\mu} h_{\rho \nu}+\nabla_{\alpha} \nabla_{\nu} h_{\rho \mu}-\nabla_{\alpha} \nabla_{\rho} h_{\mu \nu}-\nabla_{\mu} \nabla_{\alpha} h_{\rho \nu}-\nabla_{\mu} \nabla_{\nu} h_{\rho \alpha}+\nabla_{\mu} \nabla_{\rho} h_{\nu \alpha}\right)(14
\end{array}
$$




\section{References}

[1] G. Dvali, G. Gabadadze and M. Porrati, Phys. Lett. B485, 208 (2000) hep-th/0005016.

[2] C. Deffayet, Phys. Lett. B 502, 199 (2001) arXiv:hep-th/0010186.

[3] C. Deffayet, G. R. Dvali and G. Gabadadze, Phys. Rev. D 65, 044023 (2002) astro-ph/0105068.

[4] C. Deffayet, S. J. Landau, J. Raux, M. Zaldarriaga and P. Astier, Phys. Rev. D 66, 024019 (2002) arXiv:astro-ph/0201164.

[5] A. Lue, R. Scoccimarro and G. Starkman, Phys. Rev. D 69, 044005 (2004) arXiv:astro-ph/0307034. Phys. Rev. D 69, 124015 (2004) arXiv:astro-ph/0401515.

[6] D. Jain, A. Dev and J. S. Alcaniz, Phys. Rev. D 66, 083511 (2002) arXiv:astro-ph/0206224|. J. S. Alcaniz, D. Jain and A. Dev, Phys. Rev. D 66, 067301 (2002) arXiv:astro-ph/0206448.

[7] M. Ishak, A. Upadhye and D. N. Spergel, arXiv:astro-ph/0507184.

[8] E. V. Linder, arXiv:astro-ph/0507263.

[9] L. Knox, Y. S. Song and J. A. Tyson, arXiv:astro-ph/0503644.

[10] G. Dvali, A. Gruzinov and M. Zaldarriaga, Phys. Rev. D 68, 024012 (2003) arXiv:hep-ph/0212069.

[11] A. Lue and G. Starkman, Phys. Rev. D 67, 064002 (2003) arXiv:astro-ph/0212083.

[12] L. Iorio, Class. Quant. Grav. 22, 5271 (2005) arXiv:gr-qc/0504053; JCAP 0509, 006 (2005) arXiv:gr-qc/0508047; JCAP 0601, 008 (2006) arXiv:gr-qc/0510059.

[13] G. Gabadadze and A. Iglesias, Phys. Rev. D 72, 084024 (2005) arXiv:hep-th/0407049; Phys. Lett. B 632, 617 (2006) arXiv:hep-th/0508201.

[14] G. Gabadadze and A. Iglesias, arXiv:hep-th/0603199.

[15] A. Lue, arXiv:astro-ph/0510068.

[16] M. Fairbairn and A. Goobar, arXiv:astro-ph/0511029.

[17] R. Maartens and E. Majerotto, arXiv:astro-ph/0603353.

[18] K. Koyama and R. Maartens, arXiv:astro-ph/0511634. 
[19] I. Sawicki and S. M. Carroll, arXiv:astro-ph/0510364.

[20] M. A. Luty, M. Porrati and R. Rattazzi, JHEP 0309, 029 (2003) arXiv:hep-th/0303116.

[21] A. Nicolis and R. Rattazzi, JHEP 0406, 059 (2004) arXiv:hep-th/0404159.

[22] K. Koyama, arXiv:hep-th/0503191.

[23] D. Gorbunov, K. Koyama and S. Sibiryakov, arXiv:hep-th/0512097.

[24] C. Charmousis, R. Gregory, N. Kaloper and A. Padilla, arXiv:hep-th/0604086.

[25] C. Deffayet, G. R. Dvali, G. Gabadadze and A. I. Vainshtein, Phys. Rev. D 65, 044026 (2002) arXiv:hep-th/0106001.

[26] H. van Dam and M. J. G. Veltman, Nucl. Phys. B 22, 397 (1970).

V. I. Zakharov, JETP Lett. 12 (1970) 312 [Pisma Zh. Eksp. Teor. Fiz. 12 (1970) 447].

[27] A. Gruzinov, New Astron. 10, 311 (2005) arXiv:astro-ph/0112246.

[28] N. Kaloper, Phys. Rev. Lett. 94, 181601 (2005) [Erratum-ibid. 95, 059901 (2005)] arXiv:hep-th/0501028. Phys. Rev. D 71, 086003 (2005) [Erratum-ibid. D 71, 129905 (2005)] |arXiv:hep-th/0502035.

[29] N. Nakanishi, Prog. Theor. Phys. 35 (1966) 1111.

[30] B. Lautrup, Mat. Fyz. Madd. Dan. Vid. Selsk. 35 (1967) 1.

[31] A. I. Vainshtein, Phys. Lett. B 39 (1972) 393.

[32] D.R. Brill, S. Deser, Comm. Math. Phys. 32 (1973) 291.

[33] V. Moncrief, J. Math. Phys. 17 (1976) 1893.

[34] D. Kastor and J. H. Traschen, Phys. Rev. D 47, 480 (1993).

[35] A. Higuchi, Class. Quant. Grav. 8 (1991) 2023.

[36] B. Freivogel and L. Susskind, Phys. Rev. D 70, 126007 (2004) arXiv:hep-th/0408133.

[37] G. Dvali, arXiv:hep-th/0402130.

[38] G. Dvali, Talk given at the International Workshop "From Quantum to Cosmos: Fundamental Physics Research in Space", Warrenton, VA, May 22-24, 2006. 
[39] S. Deser and R. I. Nepomechie, Annals Phys. 154, 396 (1984).

S. Deser and A. Waldron, Phys. Rev. Lett. 87, 031601 (2001) arXiv:hep-th/0102166.

[40] J. Garriga and T. Tanaka, Phys. Rev. Lett. 84, 2778 (2000) arXiv:hep-th/9911055.

[41] G. Gabadadze and Y. w. Shang, arXiv:hep-th/0506040 Phys. Lett. B 635, 235 (2006) arXiv:hep-th/0511137.

[42] A. Higuchi, Nucl. Phys. B 282, 397 (1987).

[43] R. Arnowitt, S. Deser and C. W. Misner, arXiv:gr-qc/0405109.

[44] R. Dick, Class. Quant. Grav. 18, R1 (2001) hep-th/0105320; Acta Phys. Polon. B 32, 3669 (2001) hep-th/0110162.

[45] C. Deffayet and J. Mourad, Phys. Lett. B 589, 48 (2004) arXiv:hep-th/0311124. Class. Quant. Grav. 21, 1833 (2004) arXiv:hep-th/0311125.

[46] T. Shiromizu, K. i. Maeda and M. Sasaki, Phys. Rev. D 62, 024012 (2000) arXiv:gr-qc/9910076.

[47] C. Deffayet, Phys. Rev. D 66, 103504 (2002) arXiv:hep-th/0205084.

[48] C. Deffayet, Phys. Rev. D 71, 103501 (2005) arXiv:gr-qc/0412114.

[49] A. N. Aliev and A. E. Gumrukcuoglu, Class. Quant. Grav. 21, 5081 (2004) arXiv:hep-th/0407095.

[50] R. M. Wald, "General Relativity," 1984.

[51] G. Gabadadze, arXiv:hep-th/0408118.

[52] M. Porrati, Phys. Lett. B 498, 92 (2001) arXiv:hep-th/0011152.

[53] G. R. Dvali, G. Gabadadze, M. Kolanovic and F. Nitti, Phys. Rev. D 64, 084004 (2001) arXiv:hep-ph/0102216. 